\title{
Sub-sampling-based 2D localization of an impulsive acoustic source in reverberant environments
}

\author{
Muhammad Omer ${ }^{1 *}$, Ahmed A Quadeer ${ }^{1}$, Mohammad S Sharawi ${ }^{1}$ and Tareq Y Al-Naffouri ${ }^{1,2}$
}

\begin{abstract}
This paper presents a robust method for two-dimensional (2D) impulsive acoustic source localization in a room environment using low sampling rates. The proposed method finds the time delay from the room impulse response (RIR) which makes it robust against room reverberations. We consider the RIR as a sparse phenomenon and apply a recently proposed sparse signal reconstruction technique called orthogonal clustering (OC) for its estimation from the sub-sampled received signal. The arrival time of the direct path signal at a pair of microphones is identified from the estimated RIR, and their difference yields the desired time delay estimate (TDE). Low sampling rates reduces the hardware and computational complexity and decreases the communication between the microphones and the centralized location. Simulation and experimental results of an actual hardware setup are presented to demonstrate the performance of the proposed technique.
\end{abstract}

Keywords: Impulsive source localization; Room impulse response (RIR); Orthogonal clustering (OC); Sub-sampling

\section{Introduction}

Time delay estimation (TDE)-based localization methods are suitable for wideband source signals, and many algorithms [1-5] have been proposed for TDE-based localization. Such systems can be divided into two categories. The first one assumes the presence of the sound source in the far field of the microphone array. The sound wavefronts arriving at the microphones are planar, and TDE from a pair of microphones can be used to find the direction of arrival (DOA) of the signal [6]. Several DOA estimates from spatially separated microphone arrays are used to find the source location.

Another TDE-based method called the time difference of arrival (TDOA), assumes the presence of the sound source in the near field of the microphones [7-10]. The TDE from a pair of microphones is converted into range difference from which the range difference equation is formed. Multiple range difference measurements lead to multiple equations which can be solved for the unknown source position $[11,12]$. With an increased number of

*Correspondence: muhammad.omer@ucalgary.ca

1 Electrical Engineering Department, King Fahd University of Petroleum \& Minerals, Dhahran 31261, Saudi Arabia

Full list of author information is available at the end of the article microphones, the ambiguity in the source location can be resolved by the least squares solution for the multiple range difference equations.

An accurate TDE is vital for both relative angles (DOA) and range-based (TDOA) localization schemes. The most basic TDE methods are the cross-correlation (CC)-based ones [2]. The CC method assumes an ideal sound propagation model which makes it suitable for low-noise and reverberant-free outdoor environments. The improved version of the $\mathrm{CC}$ method is the generalized crosscorrelation (GCC) method [13]. The GCC is a family of algorithms which applies various weight functions to the received signal (pre-filters) in order to improve the TDE performance in noisy environment. Despite much improvement in GCC over the CC method, GCC method suffers from performance degradation in dense reverberant environments [14]. The performance of GCC method under real reverberant conditions has been analyzed in [15], and several improvements have been proposed. The fundamental problem with CC and GCC methods is their assumption that the microphones receive only the direct path signal. In [16], an adaptive eigenvalue decomposition (AED) algorithm has been proposed that assumes more realistic sound propagation models for TDE in a

\section{Springer}

(c) 2014 Omer et al.: licensee Springer. This is an Open Access article distributed under the terms of the Creative Commons Attribution License (http://creativecommons.org/licenses/by/2.0), which permits unrestricted use, distribution, and reproduction in any medium, provided the original work is properly credited. 
room reverberant environment. It first estimates the room impulse response (RIR) between the source and the pair of microphones. The TDE estimate is determined by identifying the direct paths from the two RIR. The AED algorithm for RIR estimation is a computationally complex algorithm with complexity of $\mathcal{O}\left(N^{2}\right)$. For AED to converge to the true RIR, it requires continuous snapshots of the source signal. This makes AED-based TDE unsuitable for the sources which are impulsive in nature.

In addition, these TDE techniques suffer from a time resolution problem at low sampling frequencies. The effect of under-sampling on the performance of these algorithms is discussed in [17]. The high sampling frequency requirement makes the localization process not only computationally intensive but also demands sophisticated hardware. In [5], the authors provide a brief overview, performance comparison, and problems associated with widely used TDE methods. For example, the CC method requires the acquired data to be sent to a central processing unit for TDE. This puts stress on the communication link as at high sampling rates, a large amount of data has to be communicated from the sensor to the central processing unit.

\subsection{Motivation and main objectives}

The drawbacks of the available TDE techniques motivated us in this work to find a better solution for TDE. In this work, a recently developed sparse signal reconstruction algorithm called orthogonal clustering $(\mathrm{OC})$ is applied for the TDE problem [18]. This algorithm has already been successfully implemented for impulsive noise estimation and cancellation in digital subscriber lines (DSL) [19]. The objectives of this work are as follows:

1. To tune the algorithm in [18] and apply it to enhance the robustness of an indoor acoustic source localization system against dense room reverberations.

2. To work at extremely low sampling rates to relax the computational and hardware complexity.

3. To build a hardware setup and investigate the algorithm performance in a real reverberant environment using actual measurements.

4. To benchmark the algorithm performance in terms of localization accuracy and computation time against a CC-based method.

The proposed method finds the time delays from the estimated RIR which makes it robust against room reverberations. The RIR can be considered to be a sparse phenomenon when a finite number of non-zero and temporally separated impulses (corresponding to the reflected signal components) are observed for a relatively large time interval. This sparsity assumption is utilized to estimate the RIR using OC algorithm [20]. The OC method reconstructs the sparse signals from the sub-sampled data which reduces the hardware and computational complexity. The arrival time of the direct path signal at a pair of microphones is identified from the estimated RIR, and their difference yields the desired TDE. These estimates are then used for the localization of the impulsive acoustic source using TDOA.

\subsection{Paper organization}

This paper is organized as follows: In Section 2, the signal model for RIR estimation is presented. A brief overview of sparse signal reconstruction methods and their applications in different fields is given in Section 3. The details of the OC algorithm are presented in Section 4, followed by the description of the proposed TDE method based on $\mathrm{OC}$ in Section 5. The numerical and experimental results of the TDE-based source localization utilizing the OC algorithm are presented in Section 6. A discussion on the results is given in Section 7 and the concluding remarks in Section 8.

\section{Signal model for sparse signal estimation}

TDE is a challenging task in reverberant environments. The room environment between the source-microphone pair separated by some distance can be modeled as a finite impulse response (FIR) filter with a finite response termed as the RIR. The channel taps of this filter represent the multi-path components in the received signal. From the RIR, the direct line of sight (DLOS) signal can be identified from the reflected ones. Thus, the problem of TDE is equivalent to an accurate RIR estimation at a pair of microphones and identification of the DLOS components $[5,21]$.

The signal received at the microphone due to a known excitation signal $s(t)$ can be described by a multi-path signal propagation model. The source signal $s(t)$ is assumed to be of impulsive nature with a few non-zero values for a short time duration $T$. The received signal $y(t)$ is given by

$$
y(t)=\sum_{l=0}^{L-1} \alpha_{l} s\left(t-\tau_{l}\right)+n(t),
$$

where $L$ is the number of paths capturing most of the multi-path energy, $\alpha_{l}$ and $\tau_{l}$ are the scaling magnitude factor and the time shift of path $l$, respectively, while $n(t)$ is the additive white Gaussian noise (AWGN). The discrete time representation of the model given in (1) can be compactly written as

$$
\mathbf{y}=\boldsymbol{\Phi} \alpha+\mathbf{n}
$$

where $\mathbf{y} \in \mathbb{R}^{N}$ and $\boldsymbol{\alpha} \in \mathbb{R}^{N}$ are the discrete time received and RIR vectors, respectively, and $\mathbf{n} \in \mathbb{R}^{N}$ is the AWGN 
with zero-mean and covariance matrix $\mathbf{C}_{n}=\sigma_{\mathbf{n}}^{2} \mathbf{I}$. The $N \times$ $N$ matrix $\boldsymbol{\Phi}$ is the sensing matrix whose columns consist of $N$ discretized and delayed versions of the source signal $s(t)$, i.e.,

$\boldsymbol{\Phi}=\left[\begin{array}{cccc}s(0-\Delta) & s(0-2 \Delta) & \ldots & s(0-N \Delta) \\ s(1-\Delta) & s(1-2 \Delta) & \ldots & \cdot \\ \cdot & \cdot & \cdot & \cdot \\ \cdot & \cdot & \cdot & \cdot \\ s((N-1)-\Delta) & s((N-1)-2 \Delta) & . . & s((N-1)-N \Delta)\end{array}\right]$

where $\Delta=\frac{1}{F_{S}}$ represent the time resolution with $F_{S}$ being the sampling frequency. The sampling frequency $F_{S}$ is assumed to satisfy the Nyquist criteria and thus $\Delta<<T$. The sub-sampled received signal $\mathbf{r}$ is given as

$$
\mathbf{r}=\Psi \alpha+\dot{n}
$$

where $\mathbf{r} \in \mathbb{R}^{M}$ is the sub-sampled received signal and $\mathbf{n} \in$ $\mathbb{R}^{M}$ is the AWGN of the same mean and covariance matrix as of $\mathbf{n}$. The matrix $\boldsymbol{\Psi}$ (of size $M \times N$ ) is a uniformly subsampled version of the sensing matrix $\boldsymbol{\Phi}$ where $M<<N$ and the sub-sampling ratio ${ }^{1}$ is $\frac{N}{M}=\frac{F_{S}}{F_{M}}$. As $M<<N$, (4) is an under-determined system of equations and thus is ill-posed.

The room impulse response $\boldsymbol{\alpha}$ can be assumed as a sparse signal when the length $N$ of the impulse response vector is much greater than the number of reflected signal components $L$, i.e., $N>>L$. The sparsity information of the RIR helps in its reconstruction from a small number of measurements obtained from subsampling the received signal using compressed sensing theory as discussed in the next section.

\section{Sparse signal estimation techniques}

Sparse signal reconstruction has largely been facilitated since the advent of compressed sensing (CS). As the name suggests, the scheme acquires a signal at compressed sampling rates by randomly projecting it onto a subspace much smaller than the signal dimension. Most of the naturally occurring signals are sparse in some domain, and thus, CS techniques are able to reconstruct a signal sampled at sub-Nyquist rates. This has successfully been implemented in peak-to-average power reduction in orthogonal frequency domain multiplexing (OFDM), image processing [22], impulse noise estimation and cancellation in power-line communication and DSL [19], magnetic resonance imaging (MRI) [23], channel estimation in communication systems [24], ultra-wideband (UWB) channel estimation [25], DOA estimation [26], and radar design [27].

The number of measurements $M$ are less than the number of the unknowns $N$ in (4) which renders the system of equations as under-determined. This implies that an infinite number of $\boldsymbol{\alpha}$ 's satisfy (4). This makes the problem ill-posed, but it can be solved using the sparse nature of $\alpha$. The optimal solution in this cases is to solve an $\ell_{0}$ minimization problem. The $\ell_{0}$ minimization problem is NP-hard and thus is not practical [28].

An alternate approach called convex relaxation solves a relaxed $\ell_{1}$ minimization problem by linear programming instead of $\ell_{0}$ minimization with a penalty on the number of observations. Given that the sensing matrix $\boldsymbol{\Psi}$ obeys certain properties, $\ell_{1}$ minimization approach gives accurate estimates of the sparse signal $[29,30]$. These methods do well in recovering the sparse signals from under-determined system, but at the same time, they suffer from a number of drawbacks. Some of these drawbacks are listed below:

1. Convex relaxation approaches are relatively computationally complex.

2. The structure of the sensing matrix is harmful to these methods, e.g., the Toeplitz matrix $\boldsymbol{\Phi}$ in (3). The best estimation results are obtained when the sampling is close to random.

3. They cannot make use of a priori statistical information about the signal and additive noise.

These drawbacks motivated the work in $[19,20]$ (proposed by a sub-group of the authors) to use (1) a priori statistical information, (2) the sparsity information, and (3) the structure of the sensing matrix $\boldsymbol{\Psi}$, to develop a lowcomplexity sparse signal reconstruction method which is discussed in the next section.

\section{Orthogonal clustering-based sparse signal reconstruction method}

We begin the discussion of the sparse signal reconstruction algorithm from the system model as given in (4) where the sparse signal $\alpha$ is modeled as

$$
\boldsymbol{\alpha}=\boldsymbol{\alpha}_{B} \odot \boldsymbol{\alpha}_{G}
$$

where $\odot$ denotes the Hadamard (element-by-element) multiplication. The entries of $\boldsymbol{\alpha}_{B}$ are independent and identically distributed (i.i.d.) Bernoulli random variables with success probability $p$, and the entries of $\boldsymbol{\alpha}_{G}$ are also i.i.d drawn from some zero-mean distribution with marginal probability distribution function $f(x)$. When the supports (indices with non-zero amplitude) of $\boldsymbol{\alpha}$ are known, we may write (4) as

$$
\mathbf{r}=\Psi_{S} \boldsymbol{\alpha}_{S}+\mathbf{n}
$$

where $\boldsymbol{\Psi}_{S}$ is the sub-matrix formed by the columns $\boldsymbol{\psi}_{S}$ : $\mathbf{s} \in \mathbf{S}$ indexed by the support $\mathbf{S}$. 


\subsection{Optimum estimation of $\alpha$}

The task is to obtain the optimum estimate of $\alpha$ given the observation $\mathbf{r}$. To find an optimum estimate of $\boldsymbol{\alpha}$, an minimum mean square error (MMSE) approach is pursued that can be expressed as

$$
\hat{\boldsymbol{\alpha}}^{\mathrm{MMSE}}=\sum_{S} p(\mathbf{S} \mid \mathbf{r}) \mathbb{E}[\boldsymbol{\alpha} \mid \mathbf{r}, \mathbf{S}]
$$

where the sum is evaluated over all possible supports set $\mathbf{S}$ of $\boldsymbol{\alpha}$. However, for large $N$, there will be $2^{N}$ such sets and it would become hard to evaluate this sum. The computational complexity of the estimation can be reduced by finding ways to approximate (7). In the following, the ways to calculate various terms in (7) are discussed.

\subsubsection{Evaluation of $\mathbb{E}[\alpha \mid r, S]$}

When it is known a priori that $\alpha$ conditioned on its support $\mathbf{S}$ is non-Gaussian (as the distribution of RIR is unknown), $\mathbb{E}[\boldsymbol{\alpha} \mid \mathbf{r}, \mathbf{S}]$ is hard to find and thus we replace it by the best linear unbiased estimate (BLUE),

$$
\mathbb{E}\left[\boldsymbol{\alpha}_{S} \mid \mathbf{r}\right]=\left(\boldsymbol{\Psi}_{S} \boldsymbol{\Psi}_{S}^{H}\right)^{-1} \boldsymbol{\Psi}_{S}^{H} \mathbf{r}
$$

\subsubsection{Evaluation of $p(\mathbf{S} \mid \mathbf{r})$}

The posterior probability $p(\mathbf{S} \mid \mathbf{r})$ can be obtained from the prior $p(\mathbf{S})$ using Bayes rule

$$
p(\mathbf{S} \mid \mathbf{r})=\frac{p(\mathbf{r} \mid \mathbf{S}) p(\mathbf{S})}{\sum_{S} p(\mathbf{r} \mid \mathbf{S}) p(\mathbf{S})} .
$$

According to our model, the elements of $\mathbf{x}$ are obtained from the Bernoulli process with success probability $p$, so $p(\mathbf{S})$ is given by

$$
p(\mathbf{S})=p^{|S|}(1-p)^{n-|S|} .
$$

The only probability that remains to be found is $p(\mathbf{r} \mid \mathbf{S})$.

If we consider the distribution of $\alpha \mid \mathbf{S}$ to be unknown arbitrary, then all we can say about $\mathbf{r}$ is that it is the vector in the subspace spanned by the columns of $\boldsymbol{\Psi}_{S}$, plus AWGN $n$. In this case, $p(\mathbf{r} \mid \mathbf{S})$ is given by [20]

$$
p(\mathbf{r} \mid \mathbf{S}) \sim \exp \left(-\frac{1}{\sigma_{n}^{2}}\left\|\mathbf{P}_{S}^{\perp}\right\|^{2} \mathbf{r}\right),
$$

where

$$
\boldsymbol{P}_{S}^{\perp}=\mathbf{I}-\boldsymbol{\Psi}_{S}\left(\boldsymbol{\Psi}_{S}^{H} \boldsymbol{\Psi}_{S}\right)^{-1} \boldsymbol{\Psi}_{S}^{H} .
$$

Evaluating the posterior probability and expectation over all possible supports $\left(2^{N}\right.$ such sets) requires a lot of computations. Instead, a search space can be reduced to $2^{S_{r}}$ points by finding the most probable supports $\mathbf{S}_{r}$ of $\boldsymbol{\alpha}$ using the rich structure of the problem.

\subsection{Structure-based Bayesian recovery approach}

The sensing matrix $\boldsymbol{\Phi}$ in (2) has a Toeplitz structure which is encountered in many signal processing applications of channel estimation [24], UWB channel estimation [25], and DOA estimation [26]. On the other hand, the uniformly subsampled sensing matrix $\boldsymbol{\Psi}$ of $\boldsymbol{\Phi}$ in (6) has block Toeplitz structure. The OC method exploits the structure and the impulsive nature of the source signal to estimate the RIR vector $\boldsymbol{\alpha}$ in a divide and conquer approach. The columns of a sensing matrix $\boldsymbol{\Psi}$ are not orthogonal since it is a fat matrix $(M<<N)$. However, in the aforementioned applications, a subset of columns can be found which are truly orthogonal and span the column space of $\Psi$. The columns of $\boldsymbol{\Psi}$ can be rearranged in such a way that their correlation gets lower as the columns get farther from each other. The columns of $\boldsymbol{\Psi}_{S}$ can be grouped into a maximum of $P$ orthogonal clusters, i.e., $\boldsymbol{\Psi}_{S}=\left[\boldsymbol{\Psi}_{1} \boldsymbol{\Psi}_{2} \ldots \boldsymbol{\Psi}_{P}\right]$. Equation (6) can be re-written as

$$
\mathbf{r} \mid \mathbf{S}=\left[\begin{array}{lll}
\boldsymbol{\Psi}_{1} \boldsymbol{\Psi}_{2} \ldots \boldsymbol{\Psi}_{P}
\end{array}\right]\left[\begin{array}{c}
\boldsymbol{\alpha}_{1} \\
\boldsymbol{\alpha}_{2} \\
\vdots \\
\boldsymbol{\alpha}_{P}
\end{array}\right]+\boldsymbol{n} .
$$

Notice that $|S|$ follows a binomial distribution. Its mean $N p$ can be assumed to be small and thus we can use the Poisson approximation of the binomial distribution, i.e.,

$$
P(|S|=P) \approx \frac{(N p)^{P}}{P !} \exp (-N p)
$$

We select the value of $P$ for which $P(|S|=P)<\epsilon$, where $\epsilon$ can be a very small number corresponding to the number of clusters we wish to estimate. The MMSE of $\boldsymbol{\alpha}$ in (7) can be written as

$$
\begin{aligned}
\hat{\boldsymbol{\alpha}}^{\mathrm{MMSE}} & =\left[\begin{array}{c}
\hat{\boldsymbol{\alpha}}_{1} \\
\hat{\boldsymbol{\alpha}}_{2} \\
\vdots \\
\hat{\boldsymbol{\alpha}}_{P}
\end{array}\right]=\left[\begin{array}{c}
\mathbb{E}\left[\boldsymbol{\alpha}_{1} \mid \mathbf{r}\right] \\
\mathbb{E}\left[\boldsymbol{\alpha}_{2} \mid \mathbf{r}\right] \\
\vdots \\
\mathbb{E}\left[\boldsymbol{\alpha}_{P} \mid \mathbf{r}\right]
\end{array}\right] \\
& =\left[\begin{array}{c}
\sum_{\mathbf{S}_{1}} p\left(\mathbf{S}_{1} \mid \mathbf{r}\right) \mathbb{E}\left[\boldsymbol{\alpha}_{1} \mid \mathbf{r}, \mathbf{S}_{1}\right] \\
\sum_{\mathbf{S}_{2}} p\left(\mathbf{S}_{2} \mid \mathbf{r}\right) \mathbb{E}\left[\boldsymbol{\alpha}_{2} \mid \mathbf{r}, \mathbf{S}_{2}\right] \\
\vdots \\
\sum_{\mathbf{S}_{P}} p\left(\mathbf{S}_{P} \mid \mathbf{r}\right) \mathbb{E}\left[\boldsymbol{\alpha}_{P} \mid \mathbf{r}, \mathbf{S}_{P}\right]
\end{array}\right],
\end{aligned}
$$

where $\mathbf{S}_{i}$ is the support set corresponding to the $i$ th cluster. This implies that the MMSE estimate, $\hat{\boldsymbol{\alpha}}^{\mathrm{MMSE}}$ can be obtained in a divide and conquer manner by separately evaluating the MMSE estimates $\hat{\boldsymbol{\alpha}}_{i}$ corresponding to each cluster.

\section{Proposed TDE method based on OC}

In the previous section, we presented the details of the OC method that combines a priori statistical information, sparsity, and structure of the sensing matrix to develop a fast and low-complexity sparse RIR reconstruction algorithm.

We start by finding the approximate location of dominant support by correlating the received signal with the 
sensing matrix $\Psi$. After finding the dominant supports, $P$ clusters are formed around them. This is followed by computing the likelihood of dominant supports within a cluster using (9) and $\mathbb{E}\left[\boldsymbol{\alpha}_{S} \mid \mathbf{r}\right]$ using (8). Once we have these two quantities, the MMSE estimate of $\alpha$ can be obtained by using (15). Thus, we start with an initial guess of the dominant support and refine it by exploring the supports around the initial guess. In the following, we summarize the steps involved in RIR estimation using OC algorithm:

1. Determine dominant supports. The correlation of the received signal with the columns of the sensing matrix $\boldsymbol{\Psi}$ gives us the initial guess at the regions where the dominant supports of the sparse RIR vector, $\boldsymbol{\alpha}$, might be located.

2. Form semi-orthogonal clusters. The index $i$ with the largest correlation is selected and a cluster of size $L$ is formed with the $i^{\text {th }}$ index at the center. The length of the cluster is selected on the basis of the correlation between the columns of the sensing matrix $\boldsymbol{\Psi}$. Following in this manner, we form $P$ such clusters. In a room environment considered in this work, the number of clusters are not expected to be large and thus we use $P=3$ in this scenario. The number of clusters can be increased for RIR estimation in an environment with large reverberation time.

3. Determine dominant supports within clusters. Within each cluster, the most probable supports of size $\ell=1,2, \ldots P_{c}$ are found. This is done by evaluating the likelihood of all supports of the size $\ell=1,2, \ldots P_{c}$ using (9). The expected value of $\boldsymbol{\alpha}$ given $\mathbf{r}$ is evaluated using (8). Each cluster is processed independently due to the orthogonality between the clusters. To estimate closely spaced reflections within a cluster, a high value of $P_{c}$ is selected.

4. Evaluate an estimate of $\boldsymbol{\alpha}$. The MMSE estimate of $\boldsymbol{\alpha}$ can be easily evaluated using (15) once the dominant supports for each cluster, their likelihoods, and the expected value of $\boldsymbol{\alpha}$ given $\mathbf{r}$ have been computed.

\subsection{OC-based TDE method requirements and experimental procedure}

It should be noted here that we are simply interested in estimating the time of arrival and not a perfect reconstruction of the gunshot signal. Thus, the OC method does not require the sampling frequencies $F_{S}$ or $F_{M}$ to satisfy the Nyquist criteria. Sampling the gunshot at sub-Nyquist rate is similar to sub-sampling the sensing matrix $\boldsymbol{\Phi}$ which is composed of these pulses shifted in time. In this regard, the compressed sensing theory tells us that we can subsample the signal below the Nyquist rate and still be able to reconstruct it. So, here, we are sub-sampling the signal at $F_{M}<F_{S}$ (i.e., below Nyquist criteria) while satisfying the condition that $F_{M}<\frac{1}{T}$. This is equivalent to sampling the signal at the Nyquist rate or even higher, constructing the sensing matrix $\boldsymbol{\Phi}$ using this perfectly sampled pulse, and then sampling rows of this matrix at a uniform rate. In other words, the sensing matrix constructed this way is equivalent to a sensing matrix $\boldsymbol{\Phi}$ constructed by a sub-sampled pulse.

In the following, we outline the experimental procedure involved in estimating the time delays between a pair of microphones using $\mathrm{OC}$ algorithm:

1. Each microphone acquires a signal at low data rates and transmits it to the centralized workstation. The received signal at the $i^{\text {th }}$ microphone is given by

$$
\mathbf{r}_{i}=\boldsymbol{\Psi} \boldsymbol{\alpha}_{i}+\mathbf{n}_{i}
$$

where $\mathbf{r}_{i} \in \mathbb{R}^{M}$ is the sub-sampled received signal at the $i^{\text {th }}$ microphone, $\boldsymbol{\alpha}_{i} \in \mathbb{R}^{N}$ is the impulse response of the channel between the source and $i$ th microphone, and $\dot{\mathbf{n}}_{i} \in \mathbb{R}^{M}$ is the AWGN. The matrix $\boldsymbol{\Psi}$ (of size $M \times N$ ) is a uniformly sub-sampled version of $\boldsymbol{\Phi}$ of the dictionary matrix where $M<<N$. The columns of $\boldsymbol{\Psi}$ are delayed versions of a signal which is obtained by averaging several instances of the impulsive source signals of interest at $F_{M}$, collected in an outdoor environment.

2. The RIR of each microphone $\hat{\boldsymbol{\alpha}}_{i}$ is estimated using the OC algorithm [20], as shown in (15).

$$
\hat{\boldsymbol{\alpha}}_{i}^{\mathrm{MMSE}}=\mathbb{E}\left[\boldsymbol{\alpha}_{i} \mid \mathbf{r}_{\mathbf{i}}\right]=\sum_{S} \mathbb{E}\left[\boldsymbol{\alpha}_{i} \mid \mathbf{r}_{\mathbf{i}}, \mathbf{S}\right] p\left(\mathbf{S} \mid \mathbf{r}_{i}\right)
$$

3. With the estimated impulse responses $\hat{\boldsymbol{\alpha}}_{0}$ and $\hat{\boldsymbol{\alpha}}_{1}$ at a pair of microphones, the time delay estimate is determined as the difference between the two direct paths, i.e.,

$$
\hat{\tau}=\arg \max _{l}\left|\hat{\boldsymbol{\alpha}}_{1, l}\right|-\arg \max _{l}\left|\hat{\boldsymbol{\alpha}}_{0, l}\right|
$$

4. With the multiple TDEs obtained from multiple pair of microphones, we apply conventional TDOA- or DOA-based localization method [6-10] to locate the impulsive acoustic source.

\section{Results and discussion}

In this section, the performance of the OC-based TDE method is analyzed through simulations and experiments. In the experimental part, three different configurations of the microphones will be used to compare the performance of the $\mathrm{OC}$ with $\mathrm{CC}$ algorithm in an indoor environment.

\subsection{Impulsive acoustic source}

An impulsive acoustic source considered here for simulation and experimentation is a toy gun which is capable of firing a cracker. The signal is digitally acquired in time domain using data acquisition device at a sampling rate of 

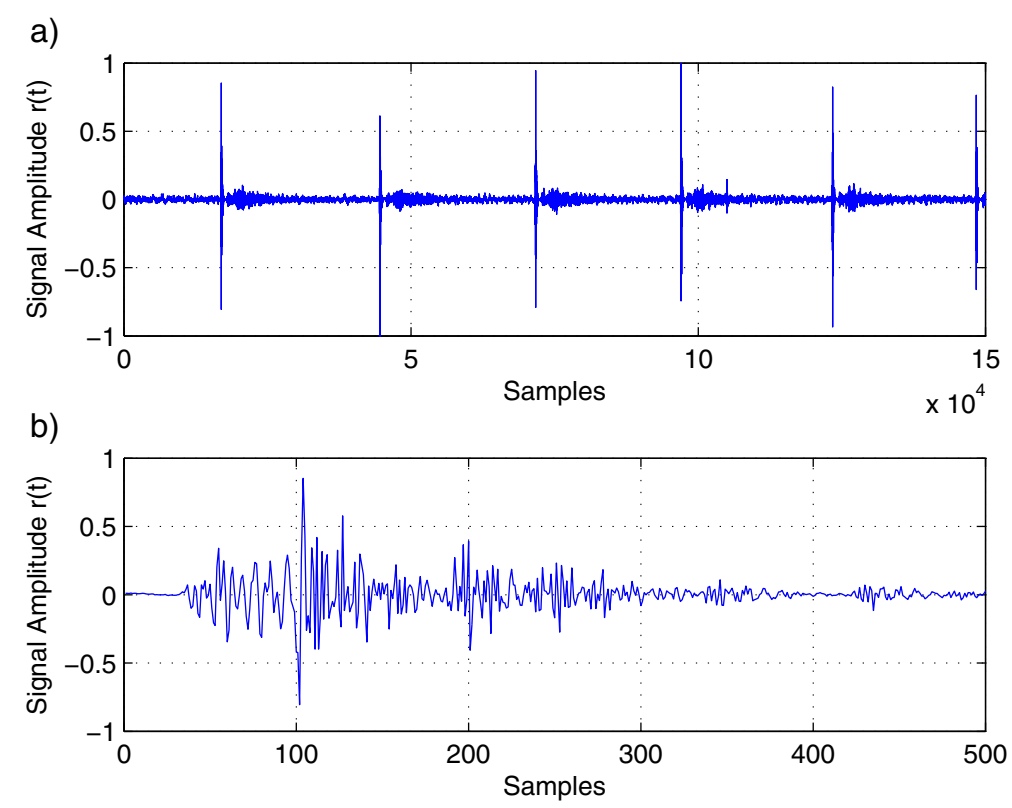

Figure 1 Impulsive acoustic source signal generated by a toy gun shot. (a) Multiple instances of an impulsive source signal generated by a toy gun fire. (b) Single instance of an impulsive source signal generated by a toy gun fire.

$30 \mathrm{kHz}$. Figure 1a shows several instances of the gunshot signal, while Figure $1 \mathrm{~b}$ zooms into one of the instances to show the signal shape.

Note that we are only concerned with $F_{M}$ (i.e., the subsampling frequency) and not with $F_{S}$. The only requirement for the proposed method to work is that $F_{M}<\frac{1}{T}$ to make sure that the gunshot is not missed completely during sub-sampling.

\subsection{TDE simulation results}

The performance of the OC-based TDE method is analyzed in simulations by creating a virtual room environment. The impulse response of the channel between a source-microphone pair placed in a room with specific dimensions is obtained using an image-source model as presented in [31]. Figure 2 shows the shoe box model of a room with dimensions $8 \times 6 \times 3 \mathrm{~m}(x \times y \times z)$.

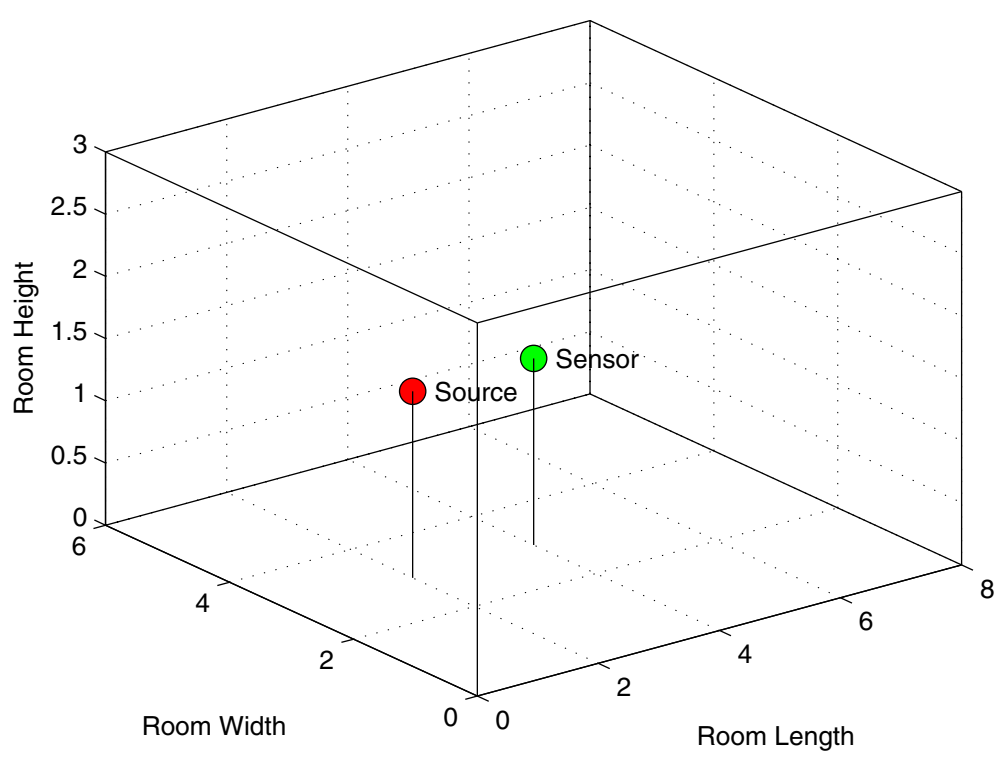

Figure 2 Shoe box room model of dimensions $8 \times 6 \times 3 \mathrm{~m}$. 


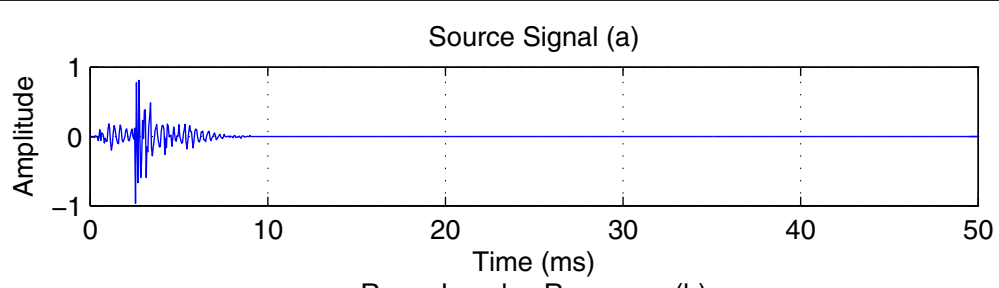

Room Impulse Response (b)

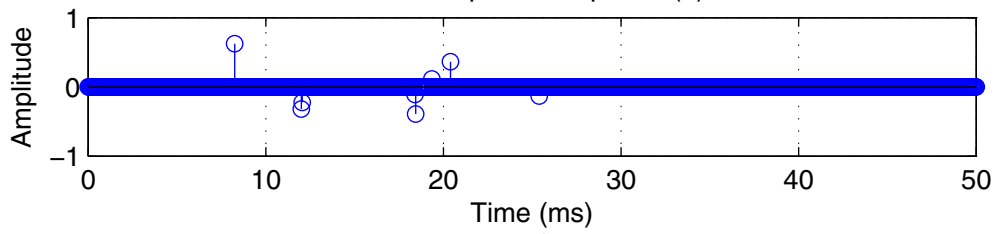

Received Signal (c)

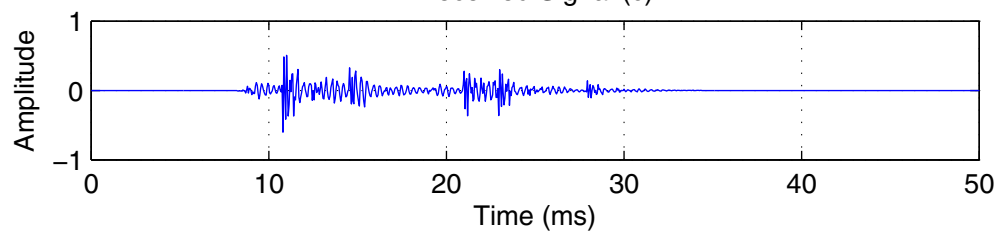

Figure 3 Typical impulsive acoustic source signal (a), room impulse response (b), and received signal at the microphone (c).

Two reverberant environments are considered with different extent of reverberations. A dense reverberant environment is created by setting high wall reflection coefficients, $\left(\left[x_{1}=0.75, x_{2}=0.75, y_{1}=0.8, y_{2}=\right.\right.$ $\left.\left.0.8, z_{1}=0.85, z_{2}=0.9\right]\right)$. For a less reverberant environment, the reflection coefficients are set low $\left(\left[x_{1}=\right.\right.$ $\left.\left.0.2, x_{2}=0.2, y_{1}=0.3, y_{2}=0.25, z_{1}=0.3, z_{2}=0.5\right]\right)$. Note that these values are selected to mimic the two extreme room environments, and any other values could have been used. The reverberation time $T_{60}$ is set to 0.25 $\mathrm{s}$, where $T_{60}$ is the time it takes for the signal energy to fall below $-60 \mathrm{~dB}$.

The sampling frequency of the microphones is initially set to $10 \mathrm{kHz}$. Figure 3a shows a typical impulsive source signal (a recorded toy gun shot). The room impulse response generated using the model in [31] is shown in Figure $3 \mathrm{~b}$. The signal received at the microphone is the convolution of source signal with the RIR as shown

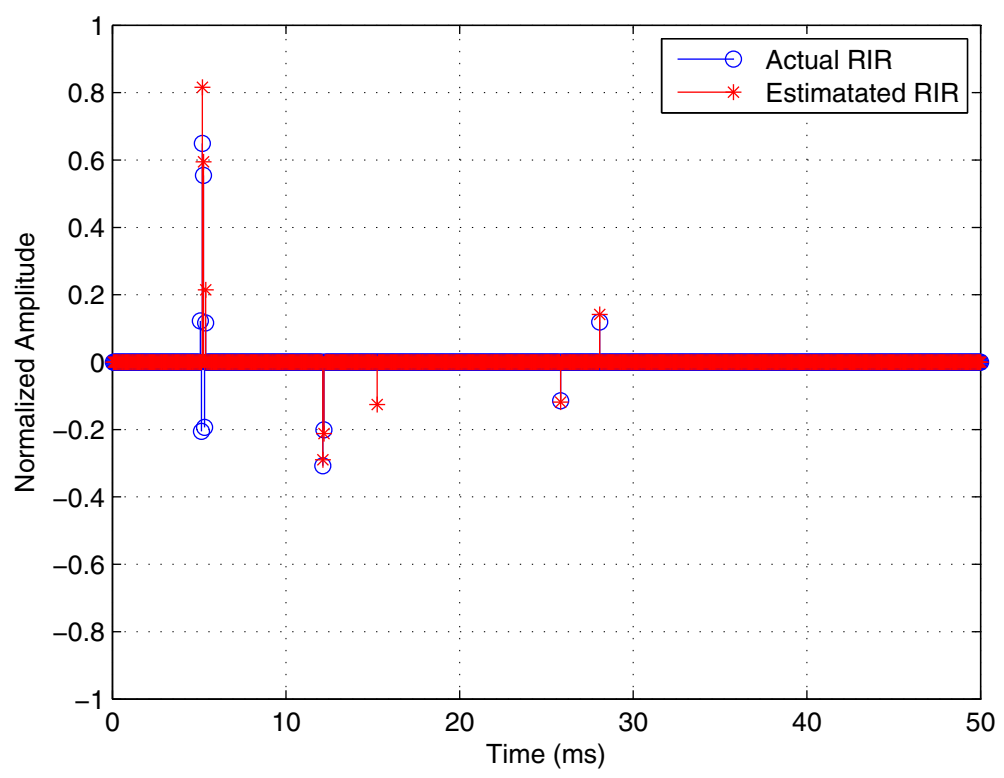

Figure 4 Example of simulated RIR estimation (low reverberations). 


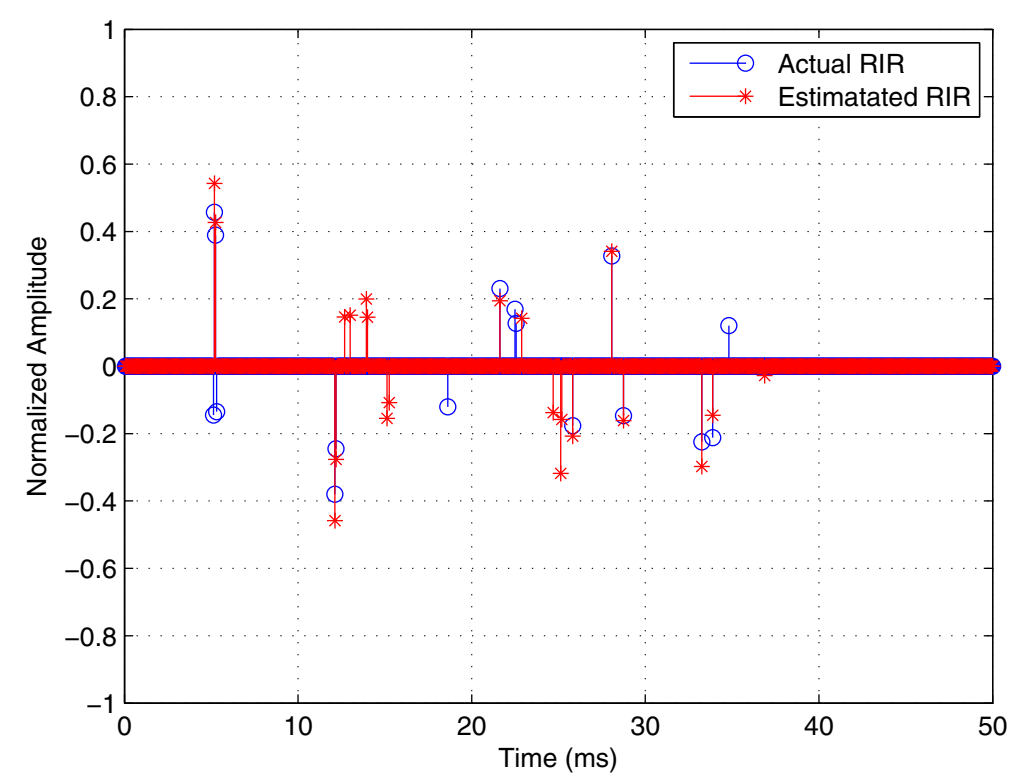

Figure 5 Example of simulated RIR estimation (dense reverberations).

in Figure 3c. The sparsity of the RIR is observable in Figure $3 b$.

An example of the RIR estimation for a low reverberant room environment is shown in Figure 4. The OC algorithm is applied for the sparse RIR estimation with sampling rate of $8 \mathrm{kHz}$. The direct path signal and most of the reflected signals are correctly estimated while few early reflections are missed. Figure 5 shows an example of a dense reverberant environment. Here, the direct path signal is accurately estimated as well as a number of early and late reflections.

Figure 6 demonstrates the performance of the OC algorithm for different sub-sampling rates. For simulation purpose, the signal-to-noise ratio (SNR) is set by adding mutually independent white Gaussian noise to the acquired microphone signal to control the SNR. The

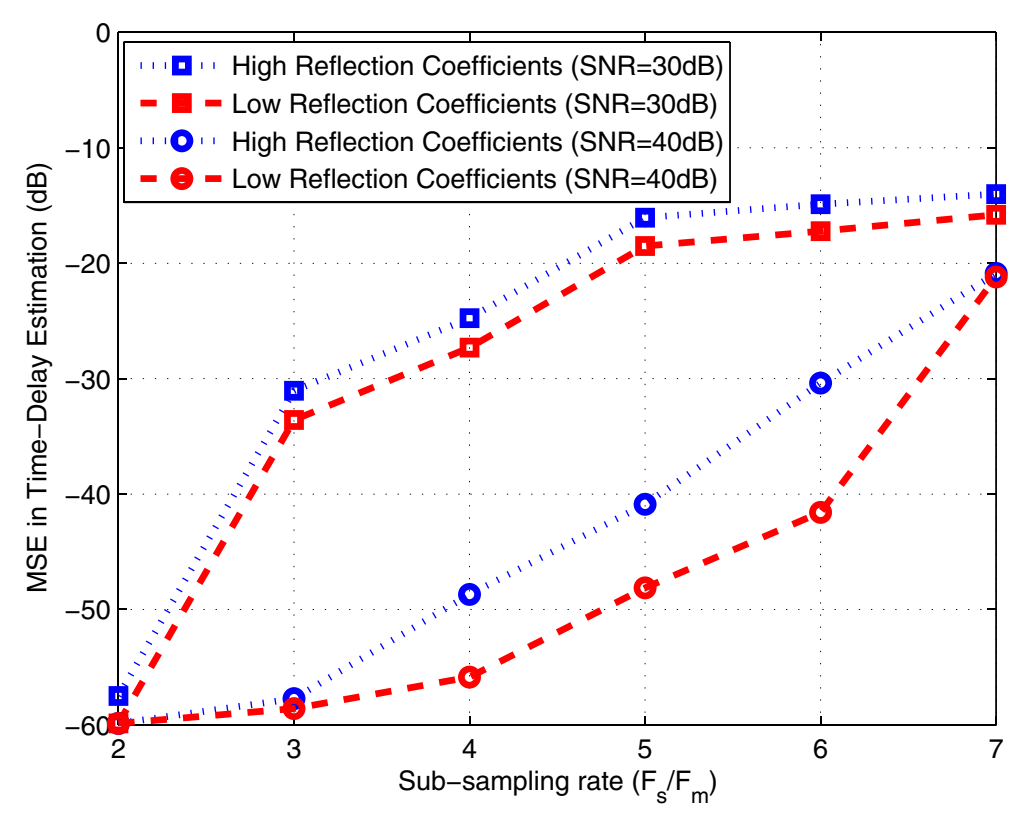

Figure 6 MSE in time delay estimation using the $O C$ algorithm for different sub-sampling rates at SNR $=30$ and $40 \mathrm{~dB}$. 


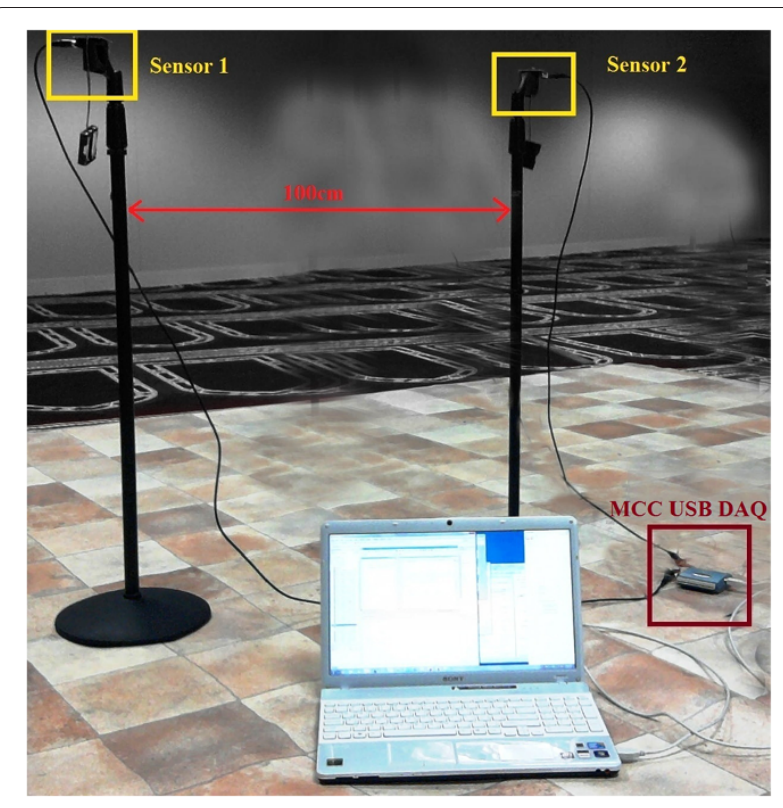

Figure 7 Experimental setup for indoor TDE.

simulation is run for 500 iterations for two values of SNR, 30 and $40 \mathrm{~dB}$, respectively. For each iteration, the position of the microphones and the acoustic source were randomly varied within the room boundaries, and RIR is generated using the image source model in [31]. It can be seen that for the 40-dB case, the MSE at low sub-sampling rates is quite low and the performance degrades gradually for higher sub-sampling rates, while there is a significant degradation in performance for sub-sampling rate greater than 2 in the $30-\mathrm{dB}$ case. With less measurements (high sub-sampling factor), the performance of the OC-based RIR reconstruction degrades which eventually results in increased MSE in TDE. Thus, the OC algorithm requires more measurements to estimate the sparse RIR at low values of SNR.

\subsection{TDE experimental results}

Figure 7 shows the actual hardware setup for TDE in a hall room of dimensions $8 \times 6 \times 3 \mathrm{~m}$. Two microphones are secured with metallic stands placed $100 \mathrm{~cm}$ apart. The electret microphones are mounted on low-noise amplifier (LNA) PCBs with a MAX 9814 LNA IC whose gain is set to $40 \mathrm{~dB}$. The output of the LNAs are connected to a 16-bit, 8-channel data acquisition (DAQ) device via audio jacks and cables. The DAQ communicates with a PC through the data acquisition tool box within MATLAB. The DAQ device is configured using MATLAB commands to acquire uniform samples of the source signal at the sampling rates of $F_{M}=16,8$, and $4 \mathrm{kHz}$, respectively. A toy gun was used as the impulsive source with a duration of approximately $10 \mathrm{~ms}$ as shown in Figure 3 . From the figure, the experimental value of the SNR is determined by taking the ratio of the signal variance $\sigma_{s}^{2}$ (portion of the waveform that corresponds to the gun shot signal) and the noise variance $\sigma_{n}^{2}$ (portion of the waveform that corresponds to the background noise received at the microphone). The SNR is determined from the following expression:

$$
\mathrm{SNR}=10 \log _{10}\left(\frac{\sigma_{s}^{2}}{\sigma_{n}^{2}}\right)
$$

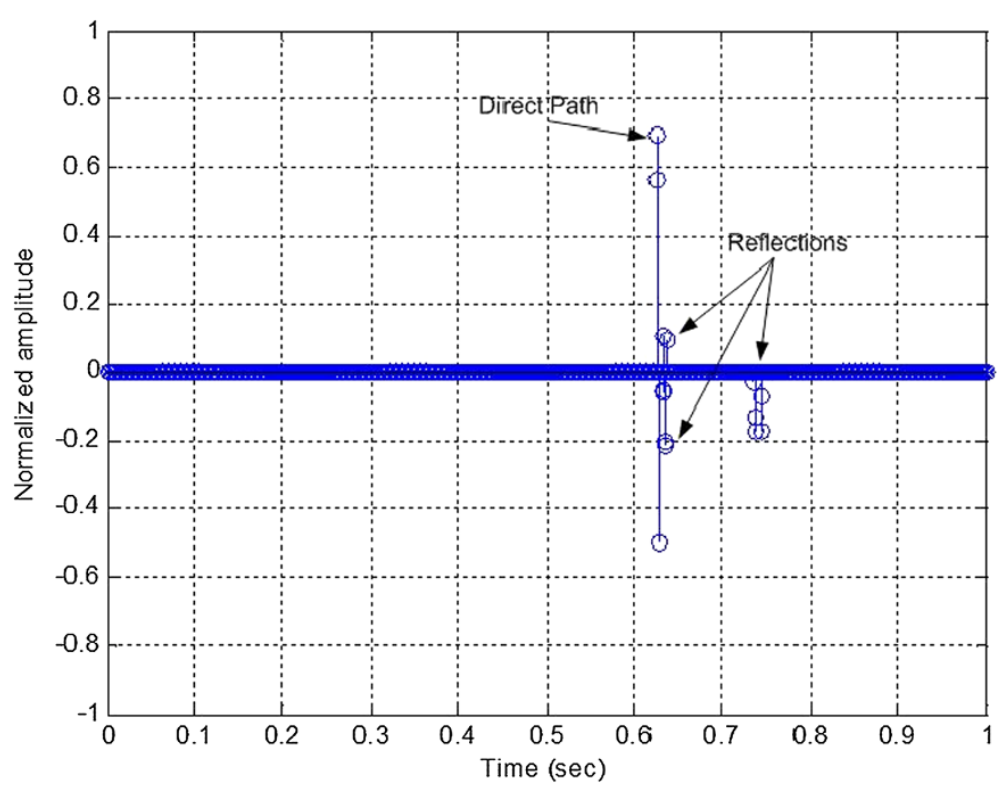

Figure 8 Experimental RIR estimate with microphone at the room center. 


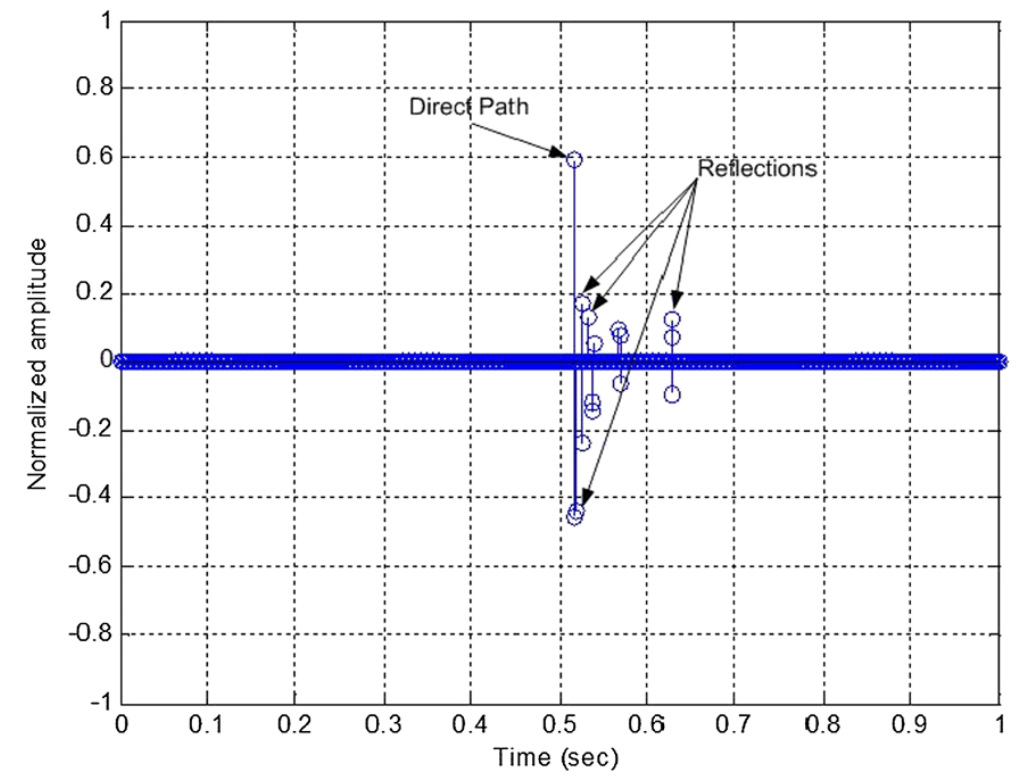

Figure 9 Experimental RIR estimate with microphone close to the wall.

The average SNR value in the experiments was SNR $\approx$ $20 \mathrm{~dB}$. The dictionary matrix $\boldsymbol{\Psi}$ in (4), required for the RIR estimation using the OC algorithm, is constructed by averaging several instances of the impulsive source signal at $F_{S}=16 \mathrm{kHz}$ where $F_{S}=F_{M}$ (i.e., sub-sampled frequency) as introduced before.

The first step in finding the time delay based on the proposed OC method is to estimate the sparse RIR. To illustrate this intermediate step of RIR estimation, we discuss two instances of the RIR estimated for different microphone positions inside the room. In the first case, the microphone is placed at the room center and with the source lying close to it. The estimated RIR shows the direct path, early reflections along with few late reflections as shown in Figure 8. Thus, we observe that the effect of room reverberations is quite low when microphone is placed away from sound obstacles (room walls). Another example of RIR estimation is conducted with the microphone placed close to the room walls. The estimated RIR contains a direct path along with a number of reflections as shown in Figure 9. The presence of the walls close to the microphone builds dense reverberations. This effect is apparent in the high number of room reflections of the estimated RIR.

The real-time functionality of the algorithm for TDE has been verified by placing the source at known locations around the microphones and acquiring the source signal at various sampling rates. Table 1 shows the time delays corresponding to three known source locations:

1. Case I: source positioned at a point on the line that passes through the two microphones.
2. Case II: source positioned close to microphone 1 , on the vertex of an isosceles triangle formed by the microphones and the source.

3. Case III: source positioned in the middle of the line joining the two microphones.

The corresponding estimated time delays using $\mathrm{CC}$ and the proposed algorithm for three sampling rate values of $F_{M}=16,8$, and $4 \mathrm{kHz}$ are shown in Table 1 . The sampling rates are selected by setting the data acquisition hardware to the desired rate. It can be seen that the proposed algorithm gives closer time delay estimates when compared to $\mathrm{CC}$ in the case of low sampling rates. This demonstrates the superior performance of the OC-based technique used

Table 1 Comparison of time delay estimates obtained using $\mathrm{CC}$ and the proposed technique based on $\mathrm{OC}$ algorithm

\begin{tabular}{ccccccc}
\hline & $\begin{array}{c}\text { Frequency } \\
(\mathbf{k H z})\end{array}$ & $\begin{array}{c}\text { True TD } \\
(\mathbf{m s})\end{array}$ & $\begin{array}{c}\text { TDE CC } \\
(\mathbf{m s})\end{array}$ & $\begin{array}{c}\text { Run time } \\
\mathbf{C C}(\mathbf{s})\end{array}$ & $\begin{array}{c}\text { TDE OC } \\
(\mathbf{m s})\end{array}$ & $\begin{array}{c}\text { Run time } \\
\text { OC (s) }\end{array}$ \\
\hline Case I & 16 & 2.941 & 2.875 & 66 & 2.875 & 70 \\
& 8 & 2.941 & 3.125 & 17 & 2.875 & 7 \\
& 4 & 2.941 & 2.250 & 4.5 & 3.312 & 3.5 \\
Case II & 16 & 1.218 & 1.250 & 66 & 1.250 & 70 \\
& 8 & 1.218 & 0.875 & 17 & 1.312 & 7 \\
& 4 & 1.218 & 1.000 & 4.5 & 1.250 & 3.5 \\
Case III & 16 & 0.000 & 0.000 & 66 & 0.000 & 70 \\
& 8 & 0.000 & 0.250 & 17 & 0.000 & 7 \\
& 4 & 0.000 & 0.750 & 4.5 & 0.187 & 3.5 \\
\hline
\end{tabular}



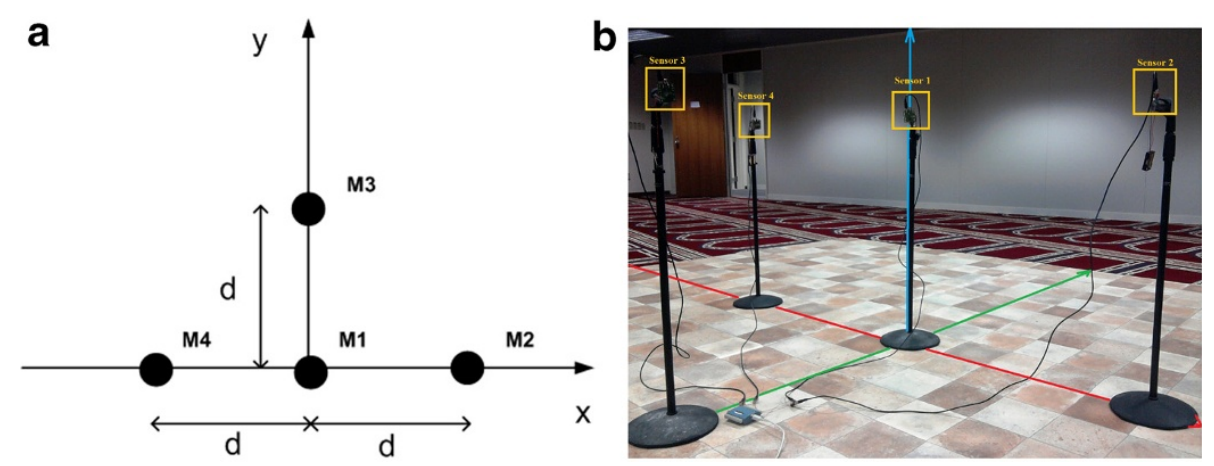

Figure 10 Microphone configuration I for source localization. (a) Configuration geometry. (b) Experimental setup.

in [19] when tuned to work for this application. In addition, the run time needed to provide TDE of the proposed algorithm are faster than $\mathrm{CC}$ at low sampling rates, which gives it a computational advantage as well.

\subsection{Source localization results}

In this sub-section, we present the results of time delaybased localization experiment. The inherent limitations of DOA-based localization method specifically for indoor environment and under low sampling rate are initially discussed. We apply the TDOA method for localization as it suits our application. Results of several localization experiments for three different microphones geometries are presented.

\subsubsection{DOA-based source localization}

The TDE is an integer multiple of the sampling period $T_{s}$ in the absence of an interpolation technique. The estimated delays suffer from poor time resolution at low sampling frequencies (large sampling period) [5]. Such low time resolution is not suitable for DOA-based localization scheme where the spatial resolution between the microphones (inter-microphone spacing) is also low. Consider a setup for DOA estimation consisting of an array of two microphones separated by a small distance $d$ and a source that lies in the far field of the array. The bearing angle $\theta$ is related to the time delay, which for $1 \mathrm{D}$ microphone array is expressed as [6]

$$
\theta=\cos ^{-1}\left(\frac{c \tau}{d}\right)
$$

where $c$ is the sound propagation velocity.

The angular resolution of an array determines the number of different DOA measurements between 0 and $\pi$. It directly corresponds to the sampling frequency $F_{s}$ (sampling time $T_{S}$ ). For instance, a microphone array, with

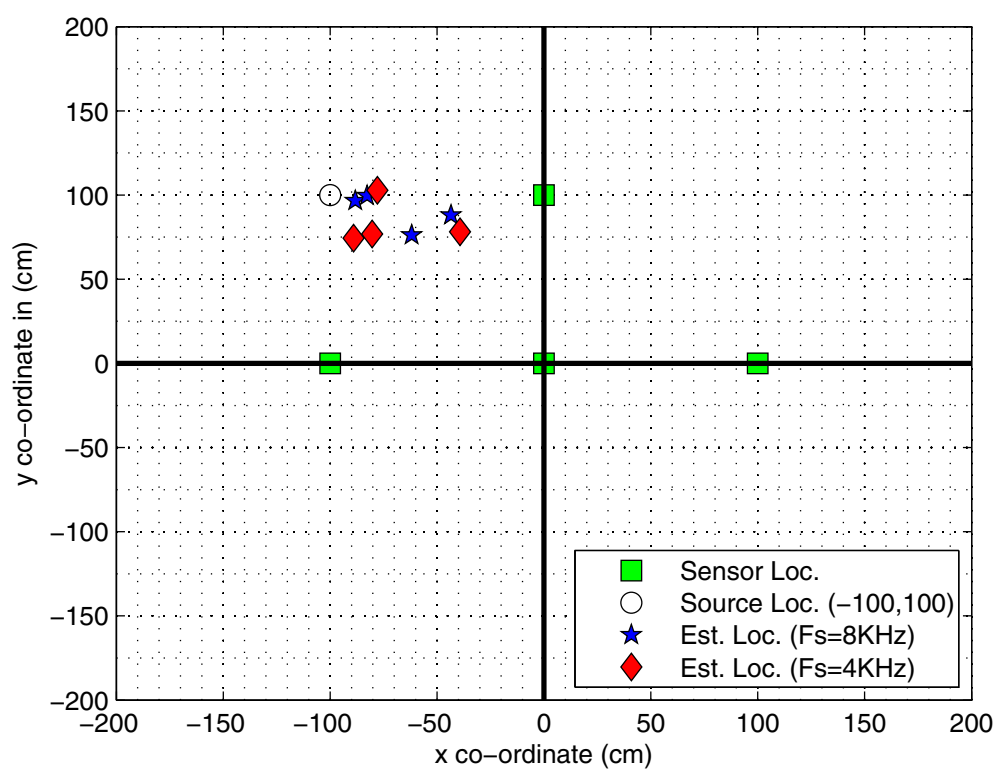

Figure 11 Microphone configuration I localization results for source at $(-100,100)$. 
a 30-cm inter-microphone spacing, can provide only 14 distinct DOA measurements at the sampling frequency $F_{s}=16 \mathrm{kHz}, 7$ measurements at $F_{s}=8 \mathrm{kHz}$ and 4 measurements at $F_{s}=4 \mathrm{kHz}$ between 0 and $\pi$, respectively.

Increasing the sampling rate improves the TDE resolution which in turn lead to a higher DOA resolution [5]. However, this approach will increase the complexity of both the TDE algorithm and hardware. Other ways to improve the DOA resolution is by increasing microphone spacing $d \mathrm{~N}$ which will increase the size of the array. Also, the far field requirement for DOA estimation makes it hard to implement in a room environment with a limited space. Moreover, large microphone spacing may cause spatial aliasing. It may also lead to higher computational complexity since more data has to be acquired for extended time durations to account for the larger delay range.

\subsubsection{TDOA-based source localization}

Time difference of arrival (TDOA) is another widely used TDE-based source localization method. The method is a two-step procedure. In the first stage, the time difference of signal arrival between a pair of microphones is estimated. With the knowledge of the propagation velocity of sound, the estimated TDOA measurement is transformed into range difference measurement from which hyperbolic

Table 2 Microphone configuration I results for the source location of $(-100,100)$

\begin{tabular}{|c|c|c|c|c|c|c|c|}
\hline $\begin{array}{l}F_{s} \\
(\mathrm{kHz})\end{array}$ & $\begin{array}{c}\text { True time } \\
\text { delay } \\
\text { (ms) }\end{array}$ & $\begin{array}{c}\text { Estimated tau } \\
\text { CC } \\
\text { (ms) }\end{array}$ & $\begin{array}{l}\text { Estimated source } \\
\text { location CC } \\
(\mathrm{cm})\end{array}$ & $\begin{array}{l}\text { Run time } \\
\text { CC } \\
\text { (s) }\end{array}$ & $\begin{array}{c}\text { Estimated tau } \\
\text { OC } \\
\text { (ms) }\end{array}$ & $\begin{array}{l}\text { Estimated source } \\
\text { location OC } \\
(\mathrm{cm})\end{array}$ & $\begin{array}{c}\text { Run time } \\
\text { OC } \\
\text { (s) }\end{array}$ \\
\hline & & 4.250 & & & 3.125 & & \\
\hline & & 1.250 & L.F. & & -0.625 & L.F. & \\
\hline & & 1.250 & & & -0.625 & & \\
\hline & & 2.125 & & & 2.375 & & \\
\hline & & -1.250 & $(-39.3,78.1)$ & & -0.937 & $(-61.8,76.2)$ & \\
\hline & & -2.250 & & & -0.375 & & \\
\hline & 2.417 & 4.500 & & & 2.375 & & \\
\hline \multirow[t]{15}{*}{8} & -1.218 & -1.125 & L.F. & 17 & -1.250 & $(-88.1,96.5)$ & 7 \\
\hline & -1.218 & -1.0000 & & & -1.000 & & \\
\hline & & 4.250 & & & 2.312 & & \\
\hline & & -1.000 & L.F. & & -1.375 & $(-82.6,99.5)$ & \\
\hline & & -7.250 & & & -0.812 & & \\
\hline & & 2.125 & & & 2.062 & & \\
\hline & & -1.500 & $(-49.7,89.0)$ & & -1.562 & $(-43.4,88.0)$ & \\
\hline & & -0.250 & & & 0.187 & & \\
\hline & & 1.250 & & & 2.250 & & \\
\hline & & -2.750 & $(-0.1,96.7)$ & & -1.500 & $(-77.9,102.7)$ & \\
\hline & & -2.500 & & & -0.687 & & \\
\hline & & 2.500 & & & 3.650 & & \\
\hline & & -1.500 & L.F. & & -1.562 & L.F. & \\
\hline & & -2.000 & & & -0.500 & & \\
\hline & 2.417 & -0.750 & & & 2.125 & & \\
\hline \multirow[t]{8}{*}{4} & -1.218 & -1.750 & $(83.3,118.8)$ & 4.5 & -1.250 & $(-39.2,78.1)$ & 3.5 \\
\hline & -1.218 & -1.250 & & & 0.312 & & \\
\hline & & 3.650 & & & 2.562 & & \\
\hline & & -0.750 & L.F. & & -0.687 & $(-89.0,74.3)$ & \\
\hline & & -1.250 & & & -1.187 & & \\
\hline & & 2.250 & & & 2.500 & & \\
\hline & & -0.500 & $(-30.2,60.0)$ & & -0.812 & $(-80.3,76.8)$ & \\
\hline & & -0.500 & & & -0.937 & & \\
\hline
\end{tabular}


range difference equation is formed. The second stage utilizes efficient algorithms to produce an unambiguous solution to the hyperbolic equations obtained from multiple microphone pairs. The solution produced by these algorithms result in the estimated source location.

The drawbacks of DOA method for indoor localization made us focus on using the TDOA-based localization method. The TDOA-based method does not make the far field assumption (i.e., plane waves arriving at microphones). Moreover, the microphones can be separated by large distances that provides higher spatial resolution for TDE at low sampling frequencies. In the following, we consider three different microphone geometries. For each geometry, we apply the CC and OC algorithms for TDE and apply the TDOA method for two-dimensional (2D) source localization.

\subsubsection{Microphone configuration I}

The microphone configuration shown in Figure 10a consists of a 2D microphone array placed in the $x y$ plane. Microphone $M 1$ placed at the center of the array is taken as a reference of the array. The location of the reference microphone $M 1$ defines the origin (intersection of $x$ and $y$ axis) of the 2D plane. Other microphones $M 2, M 3$, and $M 4$ are placed at an equidistant spacing of $d=100$ $\mathrm{cm}$ from the reference shown in Figure 10. The TDOAbased localization in $2 \mathrm{D}$ plane requires a minimum of two time delay estimates obtained from three microphones where the two TDEs corresponds to the two microphone pairs $M 1, M 2$ and $M 1, M 3$, respectively. However, adding an extra microphone $M 4$ gives a redundant time delay measurement which can be used to improve the location estimate.

The experimental setup for microphone configuration I is shown in Figure 10b. The setup is made on a $4 \times 4 \mathrm{~m}$ floor mat placed in the room center. The floor mat has a printed grid of $20 \times 20 \mathrm{~cm}$ which helps in microphones and source placement. The acoustic sensors are secured on top of metallic stands. The height of the stands form a 2D plane in which the acoustic source lies (a person firing a gun). Outputs of the pre-amplifiers are connected via audio leads and jacks to four different channels on a USB data acquisition device. MATLAB is used for data acquisition and further signal processing for the source localization algorithm.

The CC- and OC-based methods of TDE have been applied on the data acquired for different source locations. In order to assess the consistency of both algorithms, five instances of the source signal are acquired at each source location at sampling rates of 4 and $8 \mathrm{kHz}$, respectively. TDOA-based source localization is used to determine the source location based on the estimated time delays.

The estimated locations using the proposed OC method (with sub-sampling), for the source positioned at various locations in the vicinity of the acoustic sensors, were recorded for five different source locations. Figure 11 shows one of the positions where the source was at $(-100,100) \mathrm{cm}$. The figure shows the position of the microphones (filled squares), source location (hollow circles), and the estimated source locations (stars for $8 \mathrm{kHz}$ and diamond for $4 \mathrm{kHz}$ ). The plot area represents the region of the floor mat. It can be observed that the source locations for the $8-\mathrm{kHz}$ measurements lie in close proximity to the true location. The location estimates for the $4-\mathrm{kHz}$ measurements are observed to be lying relatively farther from the true source location than with the $8-\mathrm{kHz}$ measurements. This shows the trade off that exists between the accuracy of location estimates and the sub-sampling rates as shown earlier in Figure 6.

The experimental results of the CC- and OC-based time delay and source location estimates for microphone

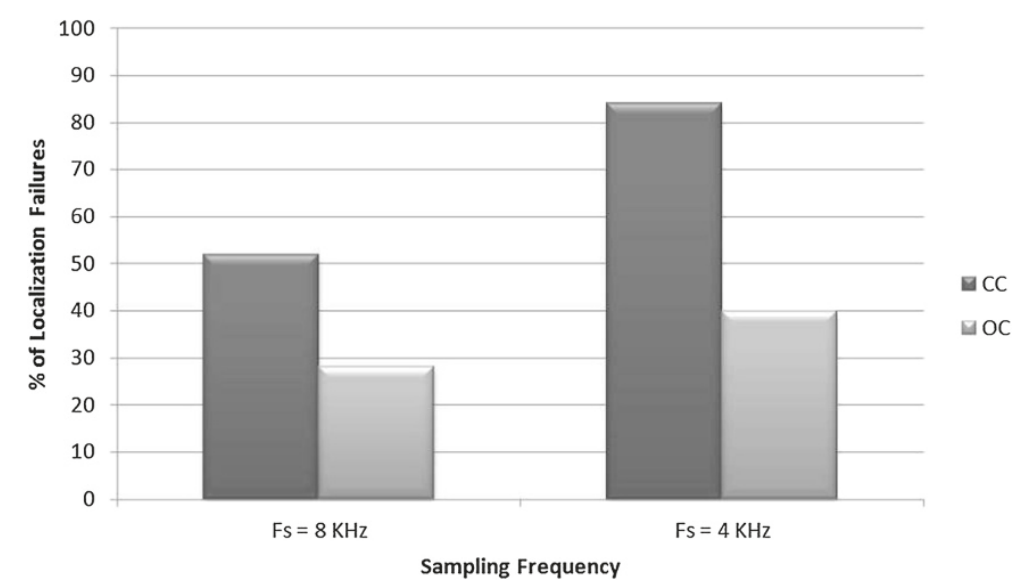

Figure 12 Localization failures in $\mathrm{CC}$ and $\mathrm{OC}$ for microphone configuration I. 

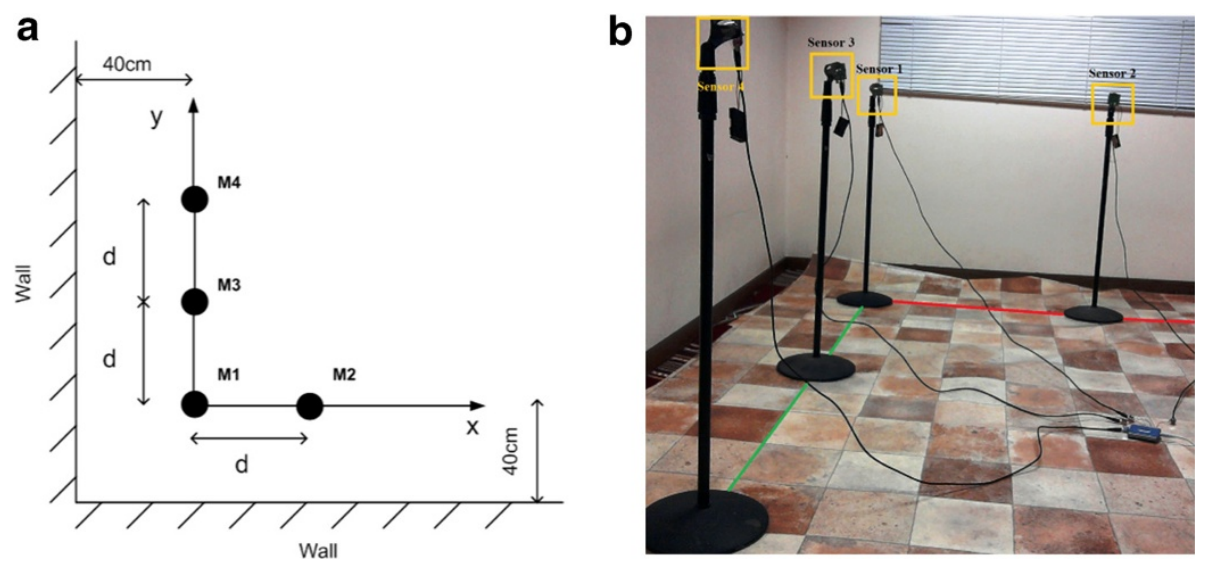

Figure 13 Microphone configuration II for source localization. (a) Configuration geometry. (b) Experimental setup.

configuration I with source location $(-100,100) \mathrm{cm}$ are also tabulated in Table 2 . The table provides the results of the estimated time delays, source location, and computation time of both TDE techniques for this specific source location sampled at two different sampling rates. Five different locations were examined for this geometry. Tables similar to Table 2 were generated for each case. From the results of this configuration, we conclude that

- The benefit of the proposed method becomes obvious when the source signal is sub-sampled. With the $8-\mathrm{kHz}$ measurements, the proposed OC-based TDE method gives better and more consistent location estimates as compared to the CC which fails (location failure (LF)) several number of times to locate the source in an acceptable region $(4 \times 4 \mathrm{~m})$. Figure 12 shows that $\mathrm{CC}$ fails $^{2}$ more than $50 \%$ of the times, while the failure rate of OC is less than $30 \%$. The OC algorithm computes TDE in $7 \mathrm{~s}$ which is much less than $17 \mathrm{~s}$ taken by CC.

- Sampling the source signal at even lower rate of $4 \mathrm{kHz}$ deteriorates the performance of the $\mathrm{CC}$ method to a much larger extent, resulting in an increased percentage of localization failures up to $85 \%$. In contrast, OC gives acceptable location estimates in many cases with relatively less percentage, $40 \%$ of localization failures. The computation time of $\mathrm{OC}$ is $3.5 \mathrm{~s}$ which is less than $4.5 \mathrm{~s}$ taken by $\mathrm{CC}$.

The acoustic source used is not an ideal point source, but in fact, it is a person exciting an acoustic source

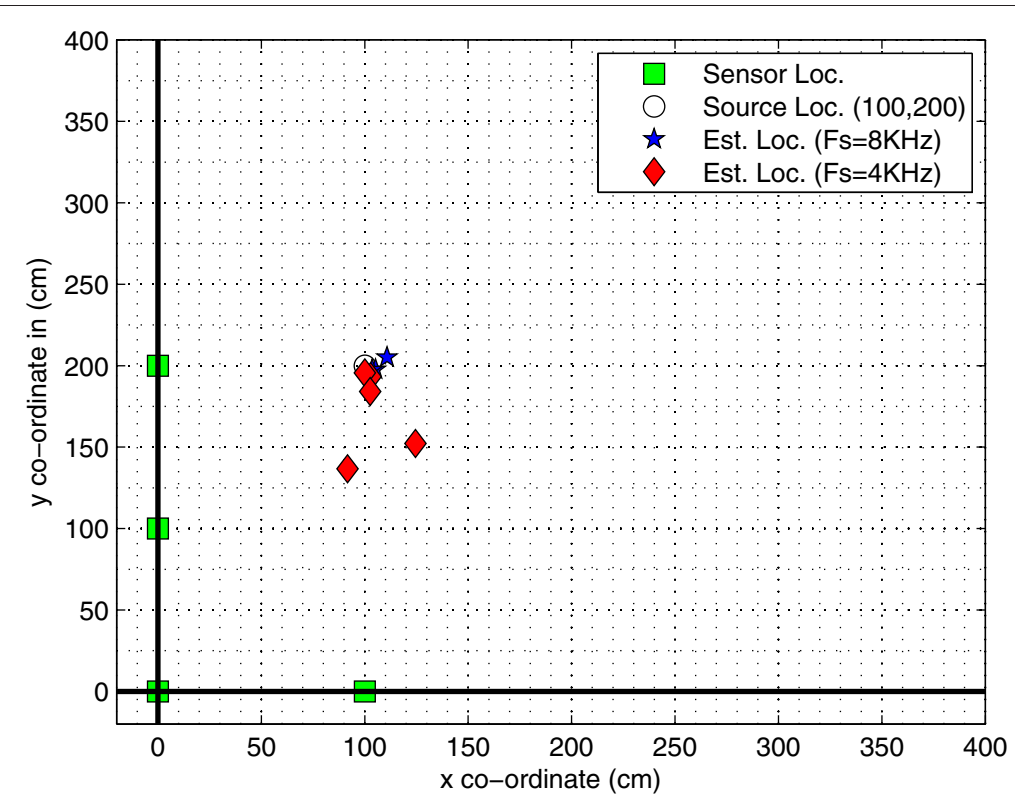

Figure 14 Microphone configuration II localization results for source at $(100,200)$. 
while standing at the true location. Hence, we expect the location estimates to lie within an area around the source $( \pm 25 \mathrm{~cm})$.

\subsubsection{Microphone configuration II}

Another microphone configuration examined for the source localization experiments is shown in Figure 13. It consists of a 2D microphone array placed in an $x y$ plane. Microphone $M 1$ is taken as an array reference and is placed at the origin. Microphones $M 2$ and $M 3$ are placed at an equidistant spacing of $d=100 \mathrm{~cm}$ from the reference along the horizontal and vertical axis, respectively. Microphone $M 4$ is placed $200 \mathrm{~cm}$ apart from the reference along the $y$ axis. The fourth microphone $M 4$ provides an extra time delay measurements which in turn improves the location estimates. This array was placed at the room corner to obtain more reverberations.

The proposed OC-based method and the CC method were applied on the acquired data for three different source locations. For each source location, the signal was acquired at sampling rates of 4 and $8 \mathrm{kHz}$, respectively. The location estimates for the source positioned at $(100,200) \mathrm{cm}$ are shown in Figure 14.

From the figure, we observe that with the sampling rate of $8 \mathrm{kHz}$, a high percentage of estimated source locations are in close proximity with the true location. Decreasing

Table 3 Microphone configuration II results for the source location of $(100,200)$

\begin{tabular}{|c|c|c|c|c|c|c|c|}
\hline $\begin{array}{c}F_{s} \\
(\mathrm{kHz})\end{array}$ & $\begin{array}{c}\text { True time } \\
\text { delay } \\
\text { (ms) }\end{array}$ & $\begin{array}{c}\text { Estimated tau } \\
\text { CC } \\
\text { (ms) }\end{array}$ & $\begin{array}{l}\text { Estimated source } \\
\text { location CC } \\
(\mathrm{cm})\end{array}$ & $\begin{array}{l}\text { Run time } \\
\text { CC } \\
\text { (s) }\end{array}$ & $\begin{array}{c}\text { Estimated tau } \\
\text { OC } \\
\text { (ms) }\end{array}$ & $\begin{array}{l}\text { Estimated source } \\
\text { location OC } \\
(\mathrm{cm})\end{array}$ & $\begin{array}{c}\text { Run time } \\
\text { OC } \\
\text { (s) }\end{array}$ \\
\hline & & 1.500 & & & -0.750 & & \\
\hline & & -5.125 & $(-10.2,157.3)$ & & -2.375 & $(105.1,197.6)$ & \\
\hline & & -3.375 & & & -3.500 & & \\
\hline & & -1.625 & & & -0.687 & & \\
\hline & & -3.375 & L.F. & & -2.562 & $(112.1,253.6)$ & \\
\hline & & -7.000 & & & -3.625 & & \\
\hline & -0.6493 & -3.375 & & & 0.562 & & \\
\hline \multirow[t]{15}{*}{8} & -2.4172 & -4.375 & L.F. & 17 & -2.750 & $(125.4,383.9)$ & 7 \\
\hline & -3.6355 & -3.875 & & & -3.562 & & \\
\hline & & -3.625 & & & -0.812 & & \\
\hline & & -2.875 & L.F. & & -2.375 & $(110.8,205.1)$ & \\
\hline & & -4.125 & & & -3.562 & & \\
\hline & & -0.250 & & & -0.750 & & \\
\hline & & -2.000 & L.F & & -2.375 & $(103.6,197.4)$ & \\
\hline & & -7.750 & & & -3.500 & & \\
\hline & & -0.750 & & & -0.750 & & \\
\hline & & 2.000 & L.F & & -2.375 & $(102.8,193.2)$ & \\
\hline & & -8.250 & & & -3.375 & & \\
\hline & & -3.250 & & & -1.250 & & \\
\hline & & -3.750 & L.F. & & -1.812 & $(124.5,152.2)$ & \\
\hline & & -4.000 & & & -1.875 & & \\
\hline & -0.6493 & 2.7500 & & & -0.687 & & \\
\hline \multirow[t]{8}{*}{4} & -2.4172 & -2.500 & $(-118.8,163.3)$ & 4.5 & -2.375 & $(100.0,195.6)$ & 3.5 \\
\hline & -3.6355 & -2.250 & & & -3.500 & & \\
\hline & & -0.750 & & & -0.750 & & \\
\hline & & 4.000 & L.F. & & -2.312 & $(102.6,184.1)$ & \\
\hline & & -4.500 & & & -3.125 & & \\
\hline & & 1.750 & & & -0.812 & & \\
\hline & & -4.750 & $(-27.5,202.1)$ & & -1.937 & $(91.6,136.7)$ & \\
\hline & & -5.250 & & & -1.562 & & \\
\hline
\end{tabular}

L.F., localization failure. 
the sampling frequency to $4 \mathrm{kHz}$, the algorithm finds close location estimates in many cases, while in others, the estimates lie a bit far from the true location. Tabulated results are shown in Table 3. We make the following observations from the results obtained:

- The close to wall microphone configuration results in an increased level of room reverberations. At $8 \mathrm{kHz}$, the proposed OC-based method is observed to be working effectively under dense room reverberation conditions. Comparing this case with the center room configuration, the localization failure has significantly decreased from $30 \%$ to $12 \%$. In contrast, the CC method suffers both from the increased level of reverberations and higher under-sampling factors. These reverberations result in an increased rate of localization failures from $50 \%$ (center room configuration) to $60 \%$. A comparison of localization failures of both $\mathrm{CC}$ and $\mathrm{OC}$ with respect to the sampling frequencies is shown in Figure 15. Moreover, the execution time of OC method is $7 \mathrm{~s}$ which is much faster than $17 \mathrm{~s}$ consumed by CC.

- At $4 \mathrm{kHz}$, the OC method performs consistently well under high sub-sampling rate with a low percentage $20 \%$ of localization failures as compared to $40 \%$ for center room configuration. On the other hand, the performance of the CC method deteriorates further at such low sampling rate. A high percentage of localization failures of more than $75 \%$ are observed with CC. The computation time of $\mathrm{OC}$ is $3.5 \mathrm{~s}$, faster than $4.5 \mathrm{~s}$ of CC.

\subsubsection{Microphone configuration III}

Figure 16 shows the third microphone configuration for the source localization experiments. It represents a $2 \mathrm{D}$ microphone array placed in an $x y$ plane. Microphone $M 1$ is taken as an array reference and is placed at the origin. Microphones $M 2$ and $M 3$ are placed at an equidistant spacing of $d=100 \mathrm{~cm}$ from the reference along the horizontal and vertical axis respectively. Microphone $M 4$ is placed $100 \mathrm{~cm}$ apart from the reference in both $x$ and $y$ axes. The fourth microphone $M 4$ provides an extra time delay measurements which in turn improves the location estimates. This geometry was also built next to the wall to increase reverberations.

The estimated locations for the source position at location $(200,100) \mathrm{cm}$ are shown in Figure 17. Table 4 shows all the experimental results conducted for microphone configuration III. From the results, we observe the following:

- At $8 \mathrm{kHz}$, the OC method works better under increased level of reverberations as less localization failure rate of $10 \%$ is observed as compared to $30 \%$ for center room configuration. Once again, we observe performance degradation of CC method with high failure rate of $\mathrm{CC}$ of $60 \%$ caused by increased reverberations and high under sampling factor. Figure 15 shows the comparison between the localization failures of OC and CC under different sampling rates. The execution time of $\mathrm{OC}$ method is $7 \mathrm{sec}$ which is much less than $17 \mathrm{~s}$ consumed by CC.

- At $4 \mathrm{kHz}$, the OC method performs effectively under dense reverberations. Comparing with center room configuration, less localization failure rate of $20 \%$ is achieved. In contrast, $\mathrm{CC}$ fails to locate the source $70 \%$ of the times due to high sub-sampling rate and increased reverberations. Figure 18 shows the rate of localization failure for both OC and CC methods under different sub-sampling rates. The computation time of $\mathrm{OC}$ is $3.5 \mathrm{~s}$, faster than $4.5 \mathrm{~s}$ of CC.

\section{Discussion}

The time delays for an indoor environment are extremely small (few milliseconds), where the source and the

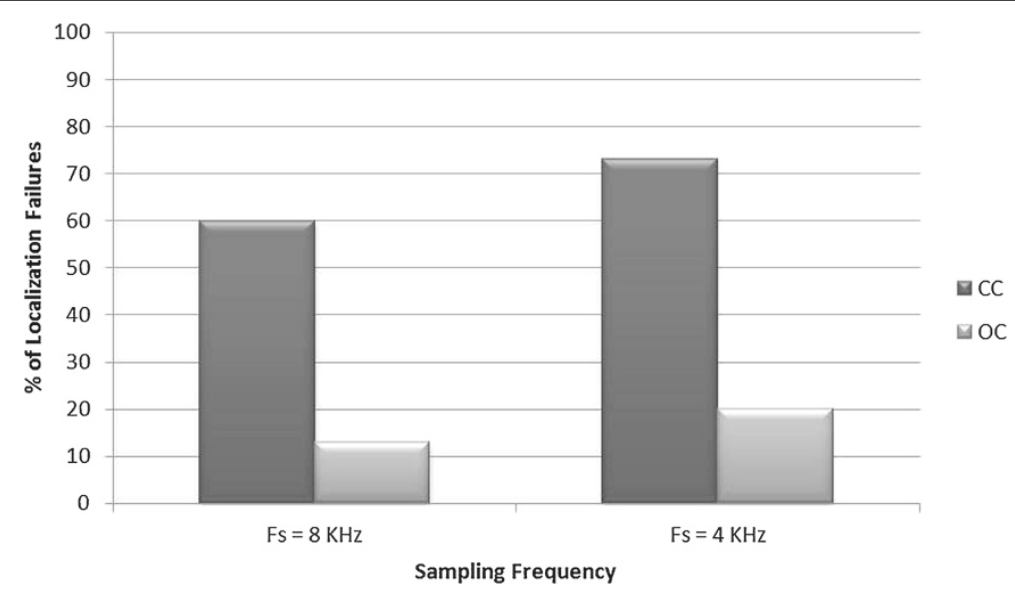

Figure 15 Localization failures in CC and OC for microphone configuration II. 

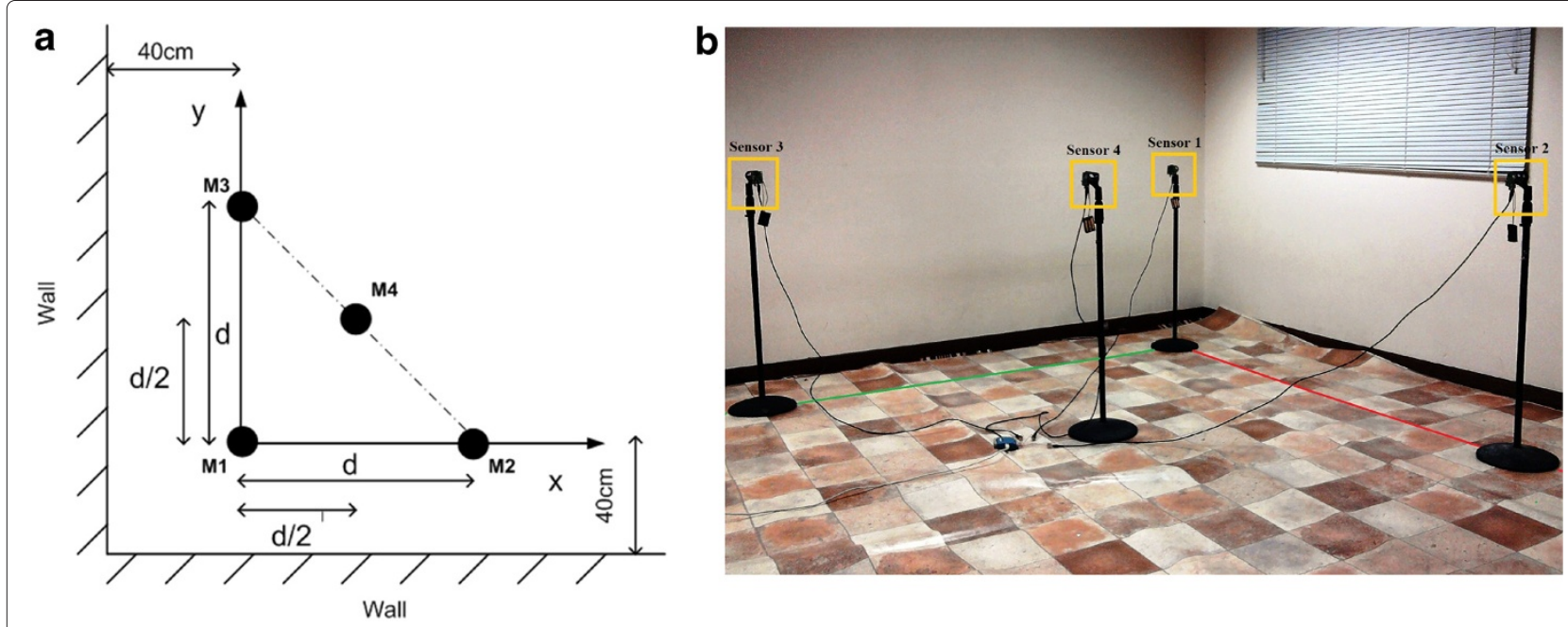

Figure 16 Microphone configuration III for source localization. (a) Configuration geometry. (b) Experimental setup.

microphones lie close to each other. In this case, accurate TDE requires high time resolution (low sampling time). The proposed method reconstructs a sparse RIR signal using signal statistics, sparsity information, and the problem structure. Although decreasing the number of measurements (sampling rate) makes the RIR estimation less accurate, this does not decrease the time resolution. In contrast, CC-based TDE method depends largely on the sampling rate. At low sampling rates, the time resolution (large sampling time) becomes poor which makes it hard to find the correlation peak close to the true time delay.
The proposed method estimates the time delays from the RIR. The accuracy of TDE depends largely on estimating the impulse which corresponds to the direct path signal. Since RIR is estimated from under-sampled signal, there is a possibility to miss the impulse corresponding to the true time delay. The TDE in such case will rely on estimating the closest reflection. Thus, increased level of reverberations in the close wall configuration favored the proposed TDE method as it suffered from low localization failures as compared to the center room configuration. On the other hand, reverberations have an adverse effect on CC method. The reflected signals cause

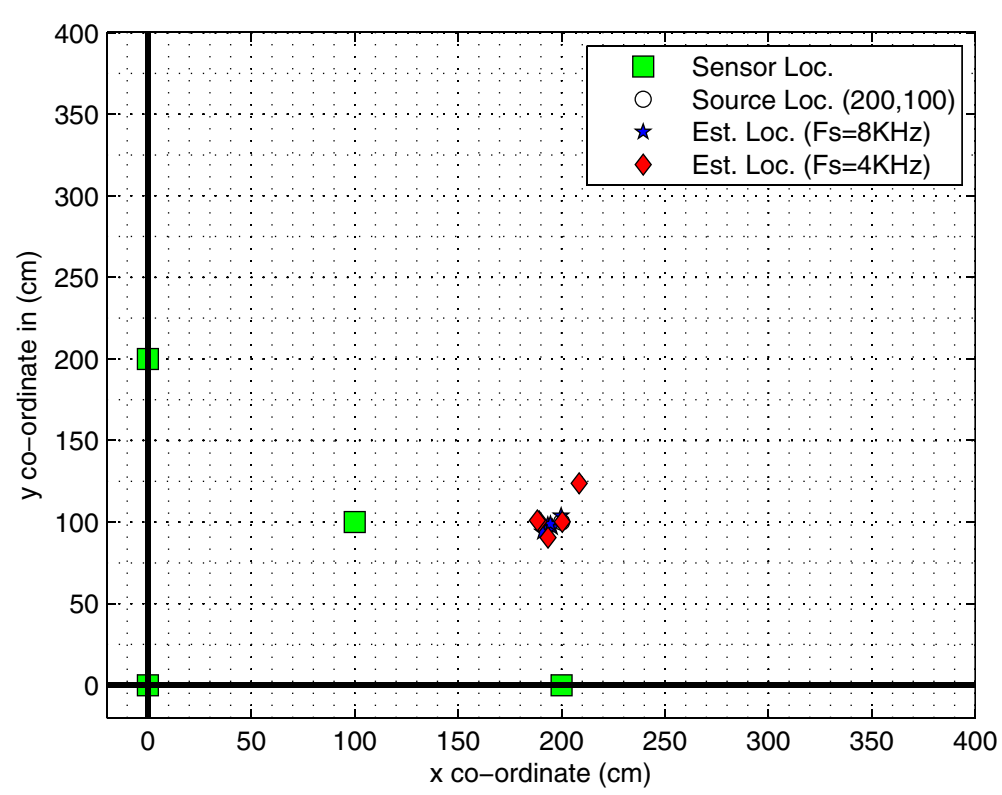

Figure 17 Microphone configuration III localization results for source at $(200,100)$. 
Table 4 Microphone configuration III results for the source location of $(200,100)$

\begin{tabular}{|c|c|c|c|c|c|c|c|}
\hline $\begin{array}{c}F_{s} \\
(\mathrm{kHz})\end{array}$ & $\begin{array}{c}\text { True time } \\
\text { delay } \\
\text { (ms) }\end{array}$ & $\begin{array}{c}\text { Estimated tau } \\
\text { CC } \\
(\mathrm{ms})\end{array}$ & $\begin{array}{c}\text { Estimated source } \\
\text { location CC } \\
(\mathrm{cm})\end{array}$ & $\begin{array}{l}\text { Run time } \\
\text { CC } \\
\text { (s) }\end{array}$ & $\begin{array}{c}\text { Estimated tau } \\
\text { OC } \\
\text { (ms) }\end{array}$ & $\begin{array}{c}\text { Estimated source } \\
\text { location OC } \\
(\mathrm{cm})\end{array}$ & $\begin{array}{c}\text { Run time } \\
\text { OC } \\
\text { (s) }\end{array}$ \\
\hline & & -5.500 & & & -3.437 & & \\
\hline & & -2.625 & L.F. & & -3.250 & $(193.3,98.3)$ & \\
\hline & & 0 & & & -0.125 & & \\
\hline & & -5.500 & & & -3.562 & & \\
\hline & & -2.000 & L.F. & & -3.625 & $(199.8,103.8)$ & \\
\hline & & -3.250 & & & -0.125 & & \\
\hline & -3.635 & -5.500 & & & -3.500 & & \\
\hline \multirow[t]{15}{*}{8} & -3.635 & -3.375 & L.F. & 17 & -3.750 & $(190.8,93.8)$ & 7 \\
\hline & 0 & 7.375 & & & 0.250 & & \\
\hline & & -5.500 & & & 3.500 & & \\
\hline & & -3.125 & $(298.3,67.4)$ & & -3.000 & $(195.5,96.7)$ & \\
\hline & & 0.625 & & & 0.187 & & \\
\hline & & -6.875 & & & -3.500 & & \\
\hline & & -3.375 & $(310.3,15.4)$ & & -3.562 & $(194.5,98.7)$ & \\
\hline & & 2.000 & & & 0 & & \\
\hline & & -5.750 & & & -3.625 & & \\
\hline & & -3.500 & L.F. & & -3.625 & $(193.4,90.4)$ & \\
\hline & & -4.250 & & & 0.312 & & \\
\hline & & -5.500 & & & -3.625 & & \\
\hline & & -3.750 & L.F. & & -3.500 & $(200.3,100.1)$ & \\
\hline & & 0.500 & & & -0.062 & & \\
\hline & -3.635 & -5.500 & & & -3.500 & & \\
\hline \multirow[t]{8}{*}{4} & -3.635 & -4.000 & L.F. & 4.5 & -4.000 & $(208.5,123.7)$ & 3.5 \\
\hline & 0 & -5.750 & & & -0.562 & & \\
\hline & & -3.250 & & & -3.312 & & \\
\hline & & -7.000 & L.F. & & -3.562 & $(189.1,100.4)$ & \\
\hline & & -2.250 & & & -0.062 & & \\
\hline & & -3.000 & & & -3.250 & & \\
\hline & & -4.250 & L.F. & & -3.312 & $(188.2,100.9)$ & \\
\hline & & -3.750 & & & -0.187 & & \\
\hline
\end{tabular}

L.F., localization failure.

multiple peaks to appear in the correlation resulting in performance degradation.

Increase in the sampling rate improved the performance of OC-based TDE method, as more measurements favored the sparse RIR signal reconstruction. Thus, OC-based localization method performed better at the sampling rate of $8 \mathrm{kHz}$ (localization failure $\leq 30 \%$ ) as compared to $4 \mathrm{kHz}$ (localization failure $\leq 40 \%$ ).

Placing the microphones close to the wall improved the performance of the proposed OC-based method. Among the three microphone configurations that we experimented, the least localization failures $(\leq 20 \%)$ were experienced with the microphone configuration II. Similar failure rate was observed in microphone configuration
III, while the center room configuration resulted in a large rate of localization failure $(\leq 40 \%)$.

\section{Conclusions}

This paper presents a novel approach for time delay estimation (TDE) for indoor impulsive source localization. The existing methods of TDE-like cross-correlation (CC) and generalized cross-correlation (GCC) suffer from performance degradation under dense reverberant environments, while the adaptive eigenvalue decomposition (AED) method for reverberant environments requires high computations due to adaptive estimation of the room impulse response (RIR). In addition, the high sampling rate requirement of the existing techniques makes 


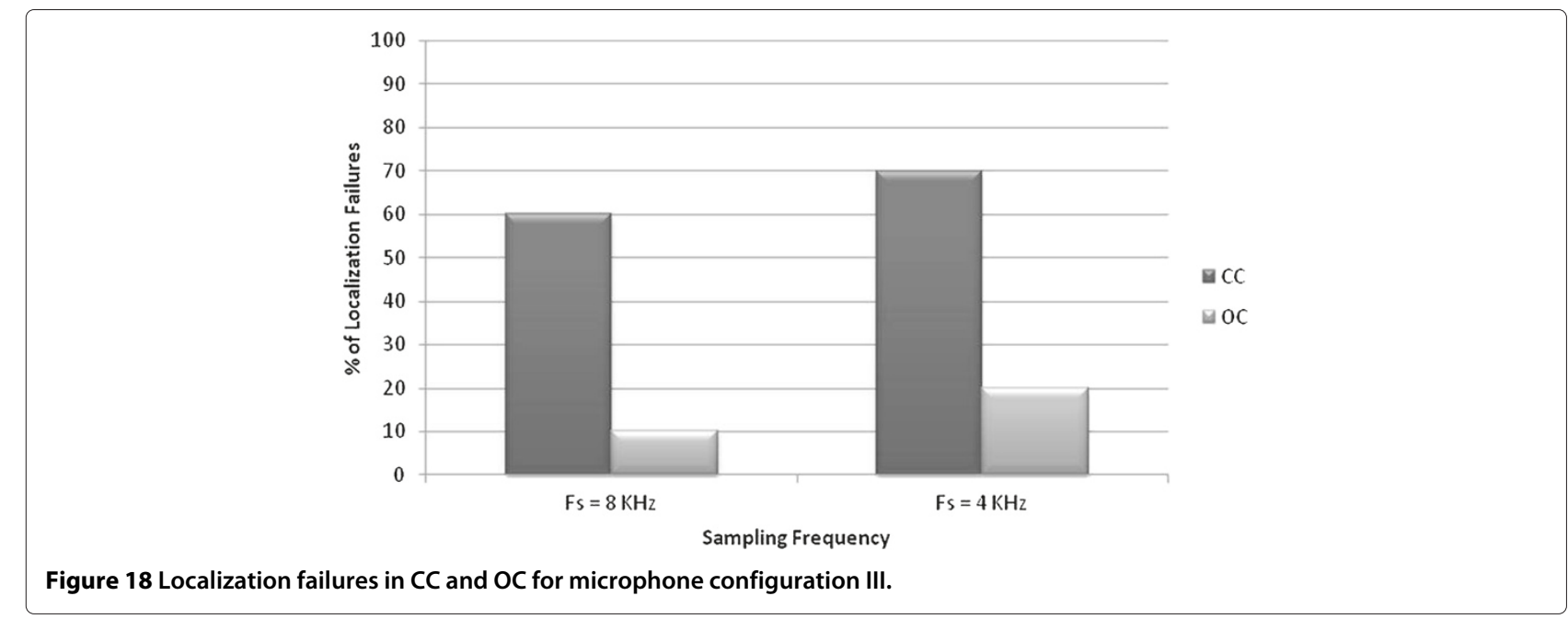

the localization process computationally intensive and imposes the need for sophisticated hardware. Moreover, the need of centralized processing in CC-based methods puts strain on the communication link between sensors and the processing unit. This motivated us to develop a more robust TDE method, based on the RIR estimation to enhance the robustness of indoor acoustic source localization. In addition, the proposed method works at extremely low sampling rates and hence decreases the computation time and hardware complexity. Moreover, the distributed nature of the proposed algorithm enables it to perform localization at the sensor level which eliminates the need for centralized processing. The performance of the proposed method for TDE and localization is analyzed by both simulations and experimental setup. The results show the improved performance of the proposed method over the existing CC method. Several microphone configurations are considered for source localization experiments. Through experimental evidences and theoretical understanding, it is found that the close wall configuration favored the proposed method.

\section{Endnotes}

${ }^{1}$ Note that it is necessary for the sub-sampling ratio to be less than $T$ to avoid missing the source signal completely, i.e., $F_{M}$ should be less than $\frac{1}{T}$. Ideally speaking, the gunshot will have frequency $\geq 20 \mathrm{kHz}$ being an acoustic signal of impulsive nature.

${ }^{2}$ Localization failure is the ratio of the number of times the algorithm fails to locate the source in an acceptable region to the total number of measurements conducted at a specific $F_{s}$.

\section{Competing interests}

The authors declare that they have no competing interests.

\section{Acknowledgements}

The authors would like to acknowledge the support provided by the King Abdulaziz City for Science and Technology (KACST) through the Science and Technology Unit at King Fahd University of Petroleum and Minerals (KFUPM) for funding this work through project no. 09-ELE763-04 as part of the National Science, Technology and Innovation Plan.

\section{Author details}

${ }^{1}$ Electrical Engineering Department, King Fahd University of Petroleum \& Minerals, Dhahran 31261, Saudi Arabia. ${ }^{2}$ Electrical Engineering Department, King Abdullah University of Science and Technology (KAUST), Thuwal 23955, Saudi Arabia.

Received: 22 December 2013 Accepted: 26 June 2014

Published: 22 July 2014

\section{References}

1. G Carter, A Nuttall, P Cable, The smoothed coherence transform. Proc. IEEE. 61, 1497-1498 (1973)

2. J lanniello, Time delay estimation via cross-correlation in the presence of large estimation errors. IEEE Trans. Acoustics Speech Signal Process. 30, 998-1003 (1982)

3. G Jacovitti, G Scarano, Discrete time techniques for time delay estimation. IEEE Trans. Signal Process. 41, 525-533 (1993)

4. MS Brandstein, Time-delay estimation of reverberated speech exploiting harmonic structure. J. Acoust. Soc. Am. 105, 2914 (1999)

5. J Chen, J Benesty, YA Huang, Time delay estimation in room acoustic environments an overview. EURASIP J. Adv. Signal Process. 2006, $1-20$ (2006)

6. B Berdugo, MA. Doron, J Rosenhouse, H Azhari, On direction finding of an emitting source from time delays. J. Acoust. Soc. Am. 105, 3355 (1999)

7. U Klee, T Gehrig, J McDonough, Kalman filters for time delay of arrival-based source localization. EURASIP J. Adv. Signal Process. 2006 $1-16(2006)$

8. PB Muanke, C Niezrecki, Manatee position estimation by passive acoustic localization. J. Acoust. Soc. Am. 121, 2049-59 (2007)

9. Y Teshima, J-y Takayama, S Ohyama, K Oshima, Person localization using TDOA of non-speech sound signal based on multiplexed CSP analysis, in Proceedings of SICE Annual Conference 2010 (Taipei, 18-21 Aug 2010)

10. THan, X Lu, Q Lan, Pattern recognition based Kalman filter for indoor localization using TDOA algorithm. Appl. Math. Model. 34, 2893-2900 (2010)

11. Y Chan, $\mathrm{KHo}$, A simple and efficient estimator for hyperbolic location. IEEE Trans. Signal Process. 42, 1905-1915 (1994)

12. P Stoica, J Li, Lecture notes - source localization from range-difference measurements. IEEE Signal Process. Mag. 23, 63-66 (2006) 
13. C Knapp, G Carter, The generalized correlation method for estimation of time delay. IEEE Trans. Acoustics Speech Signal Process. 24, 320-327 (1976)

14. B Champagne, S Bedard, A Stephenne, Performance of time-delay estimation in the presence of room reverberation. IEEE Trans. Speech Audio Process. 4, 148-152 (1996)

15. J Chen, J Benesty, YA Huang, Performance of GCC- and AMDF-based time-delay estimation in practical reverberant environments. EURASIP J. Adv. Signal Process. 2005, 25-36 (2005)

16. J Benesty, Adaptive eigenvalue decomposition algorithm for passive acoustic source localization. The J. Acoust. Soc. Am. 107, 384-392 (2000)

17. OM Bouzid, GY Tian, J Neasham, B Sharif, Investigation of sampling frequency requirements for acoustic source localisation using wireless sensor networks. Appl. Acoustics. 74(2), 269-274 (2013)

18. AA Quadeer, SF Ahmed, TY Al-Naffouri, Structure based Bayesian sparse reconstruction using non-Gaussian prior, in 49th Annual Allerton Conference on Communication, Control, and Computing (Allerton) (Monticello, Sept 2011), pp. 277-283

19. TY Al-Naffouri, AA Quadeer, G Caire, Impulsive noise estimation and cancellation in DSL using orthogonal clustering, in 2011 IEEE International Symposium on Information Theory Proceedings (St. Petersburg, Aug 2011), pp. 2841-2845

20. AA Quadeer, TY Al-Naffouri, Structure-based Bayesian sparse reconstruction. IEEE Trans. Signal Process. 60, 6354-6367 (2012)

21. R Ratnam, DL Jones, BC Wheeler, WD O'Brien, CR Lansing, AS Feng, Blind estimation of reverberation time. J. Acoust. Soc. Am. 114, 2877-92 (2003)

22. M Duarte, M Davenport, D Takhar, J Laska, K Kelly, R Baraniuk, Single-pixel imaging via compressive sampling. IEEE Signal Process. Mag. 25, 83-91 (2008)

23. M Lustig, D Donoho, JM Pauly, Sparse MRI: The application of compressed sensing for rapid MR imaging. Magn. Reson. Med. 58, 1182-95 (2007)

24. J Haupt, WU. Bajwa, G Raz, R Nowak, Toeplitz compressed sensing matrices with applications to sparse channel estimation. IEEE Trans. Inf. Theory. 56, 5862-5875 (2010)

25. JL Paredes, GR Arce, Z Wang, Ultra-wideband compressed sensing: channel estimation. IEEE J. Selected Top. Signal Process. 1, 383-395 (2007)

26. G Leus, A Pandharipande, Direction estimation using compressive sampling array processing, in 2009 IEEE/SP 15th Workshop on Statistical Signal Processing (Cardiff, Sept 2009), pp. 626-629

27. M Herman, T Strohmer, High-resolution radar via compressed sensing IEEE Trans. Signal Process. 57, 2275-2284 (2009)

28. EJ Candès, JK Romberg, T Tao, Stable signal recovery from incomplete and inaccurate measurements. Commun. Pure Appl. Math. 59, 1207-1223 (2006)

29. J Tropp, Just relax: convex programming methods for identifying sparse signals in noise. IEEE Trans. Inf. Theory. 52, 1030-1051 (2006)

30. EJ Candes, PA Randall, Highly robust error correction by convex programming. IEEE Trans. Inf. Theory. 54, 2829-2840 (2008)

31. EA Lehmann, AM Johansson, Prediction of energy decay in room impulse responses simulated with an image-source model. J. Acoust. Soc. Am. 124, 269-77 (2008)

doi:10.1186/1687-6180-2014-116

Cite this article as: Omer et al.: Sub-sampling-based 2D localization of an impulsive acoustic source in reverberant environments. EURASIP Journal on Advances in Signal Processing 2014 2014:116.

\section{Submit your manuscript to a SpringerOpen ${ }^{\circ}$ journal and benefit from:}

- Convenient online submission

- Rigorous peer review

- Immediate publication on acceptance

- Open access: articles freely available online

- High visibility within the field

- Retaining the copyright to your article

Submit your next manuscript at $\boldsymbol{\wedge}$ springeropen.com 